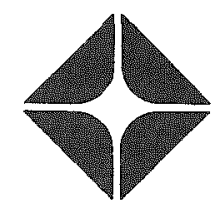

\title{
RADIOACTIVITY IN GASEOUS WASTE DISCHARGED FROM THE SEPARATIONS FACILITIES DURING 1975
}

J. D. Anderson

March 12, 1976

PREPARED FOR THE U.S. ENERGY RESEARCH AND DEVELOPMENT ADMINISTRATION UNOER CONTRACT E (AS-I) 2130

\section{PRELIMINARY REPORT}

THIS REPORT CONTAINS INFORMATION OF APRELIMINARY NATURE. IT IS SUBJECT TO REVISION OR CORRECTION AND THEREFORE DOES NOT REPRESENT A FINAL REPORT. IT WAS PREPARED PRIMARILY FOR INTERNAL USE WITHIN THE ATLANTIC RICHFIELDHANFORD COMPANY ANY EXPRESSED VIEWS AND OPINIONS ARE THOSE OF THE AUTHOR AND NOT NECESSARILY OF THE COMPANY.

\section{NOTICE}

THIS REPORT WAS PREPARED AS AN ACCOUNT OF WORK SPONSORED $B Y$ THE UNITED STATES GOVERNMENT. NEITHER THE UNITED STATES NOR THE UNITED STATES ENERGY RESEARCH AND DEVELOPMENT ADMINISTRATION, NOR ANY OF THEIR EMPLOYEES. NOR ANY OF THEIR CONTRACTORS. SUBCONTRACTORS OR THEIR EMPLOYEES, MAKES ANY WARRANTY, EXPRESS OR IMPLIED, OR ASSUMES ANY LEGAL LIAE ILITY OR RESPONSIEILITY FOR THE ACCURACY, COMPLETENESS OR USEFULNESS OR ANY INFORMATION, APPARATUS, PRODUCT OR PROCESS DISCLOSED, OR REPRESENTS THAT ITS USE WOULD NOT INFRINGE PRIVATELY OWNED RIGHTS. 


\section{DISCLAIMER}

This report was prepared as an account of work sponsored by an agency of the United States Government. Neither the United States Government nor any agency Thereof, nor any of their employees, makes any warranty, express or implied, or assumes any legal liability or responsibility for the accuracy, completeness, or usefulness of any information, apparatus, product, or process disclosed, or represents that its use would not infringe privately owned rights. Reference herein to any specific commercial product, process, or service by trade name, trademark, manufacturer, or otherwise does not necessarily constitute or imply its endorsement, recommendation, or favoring by the United States Government or any agency thereof. The views and opinions of authors expressed herein do not necessarily state or reflect those of the United States Government or any agency thereof. 


\section{DISCLAIMER}

Portions of this document may be illegible in electronic image products. Images are produced from the best available original document. 


\section{RADIOACTIVITY IN GASEOUS WASTE DISCHARGED \\ FROM THE SEPARATIONS FACILITIES \\ DURING 1975}

J. D. Anderson

Waste Handling and Plutonium Finishing Process Engineering Engineering Department

Production and Waste Management Division

March 12, 1976

Operated for the Energy Research and Development Administration by Atlantic Richfield Hanford Company under Contract E(45-1)-2130 


\section{CONTENTS}

PAGE

INTRODUCTION

SUMMARY

1

DISCUSSION

SUMMARY OF RAOIOACTIVE GAS OISCHARGEDTO TME ATMOSPHERE OURING 1975

RADI OACTIUE GASEOUS WASTE - MONTHLY DATA - 1975

\begin{tabular}{|c|c|}
\hline $291-4-1$ & PUREX PROCESS AND CANYON \\
\hline $296-1$ & PUREX N AND O CELLS AND PR ROOM \\
\hline $296-A-2$ & PUREX WEST SAMPLE GALERY HOOOS \\
\hline $296-4-3$ & PUREX EAST SAMPLE GMLERY HOOOS \\
\hline $296-A-5$ & PUREX LA HOODS AND GLOVE BOXES \\
\hline $296-A-5$ & PUREX EAST SAMPLE GALLERY ANO U CELL \\
\hline $296-A-7$ & PUREX WEST SAMPLE GMLLER AND R CELL \\
\hline $296-2=8$ & PUREX P AND O GALLEFY WHITE ROOM \\
\hline $296-A-10$ & PUREX GURIAL TUNNEL NO. 2 \\
\hline $296-A-12$ & AR VAULT VESS:L VENT \\
\hline $296-A-13$ & AR VAULT CANYON AND CELL \\
\hline $296-1-14$ & 293-A BUILOING \\
\hline $296-A-17$ & 241-A, AX, ANO AY TANK FARM VENT \\
\hline $296-A-18$ & 111-AY TANK ANVULUS \\
\hline
\end{tabular}

2

2

3

3

4

4

5

5

6

6

7

7

6 
CONTENS

PAGE

$$
\begin{aligned}
& 296-A=19 \\
& 291-B-1 \\
& 296-B-2 \\
& 296-B=3 \\
& 296-B=4 \\
& 296-B-5 \\
& 296-P=10 \\
& 291-C=1 \\
& 296-C-2 \\
& 296-C=5 \\
& 296-P=1 \\
& 296=P=2 \\
& 296-P=3 \\
& 296-P=4 \\
& 296-P=5 \\
& 296-P-6 \\
& 296-P=7 \\
& 296-P=8 \\
& 296-P=9
\end{aligned}
$$

102 - AY TAMK ANNULUS

B PLANT PROCESS AND CANYON

IN-TAKK SOLIDIFICATION NO. 1

IN-TAN SOLIDIF ICATION NO. 2

10

221 - BB VESSEL VENT NO. 3

11

$221-98$ BUILDING

11

225-BUILOING

12

SEMIWORKS PROCESS NO CELLS

12

13

13

14

14

15

15

ITS gOTTOMS THKS

16

105 ANO 106-C TANKS

16

17

ITS BOTTOHS TANS

47

TX FARM BOTTONS TANKS

16 


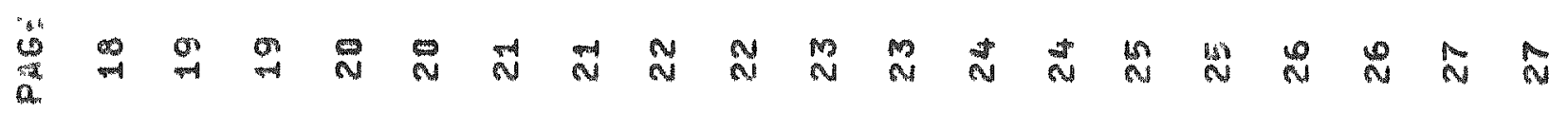

$\frac{1}{11}$

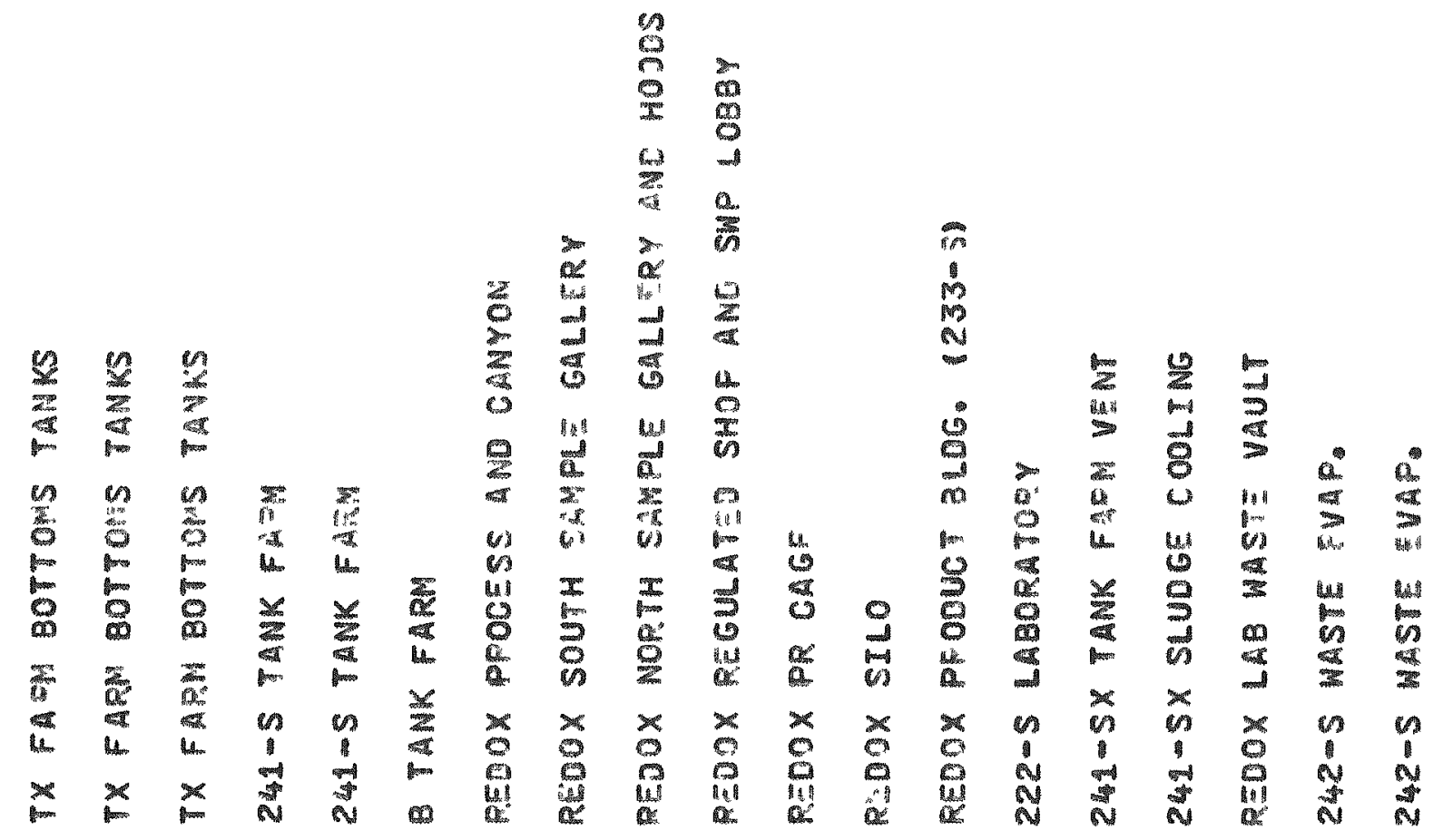

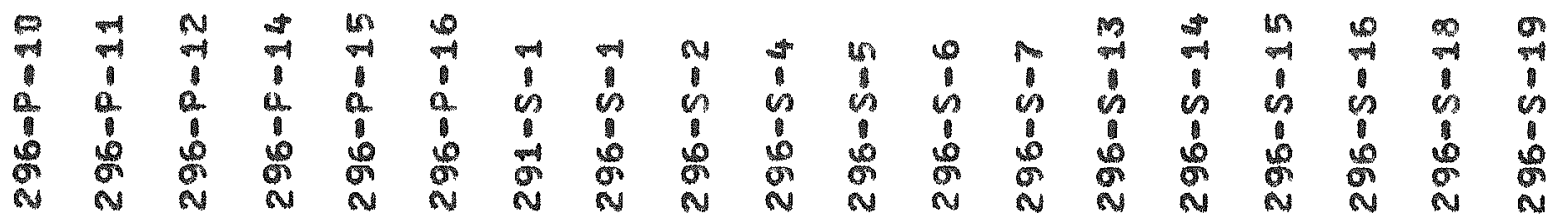




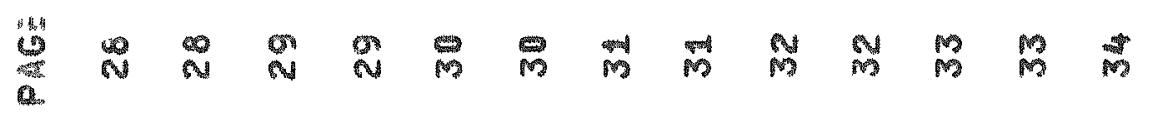

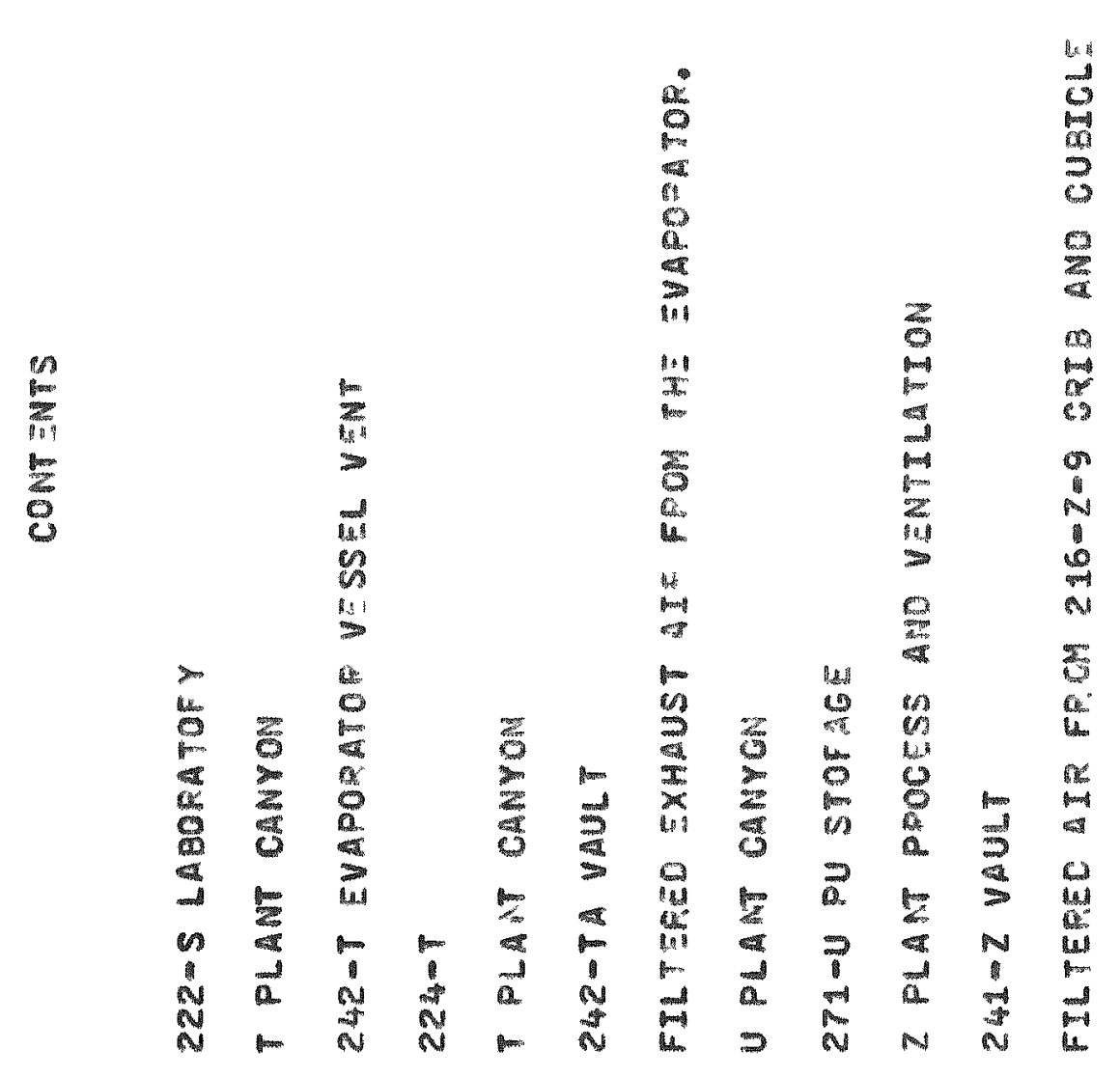

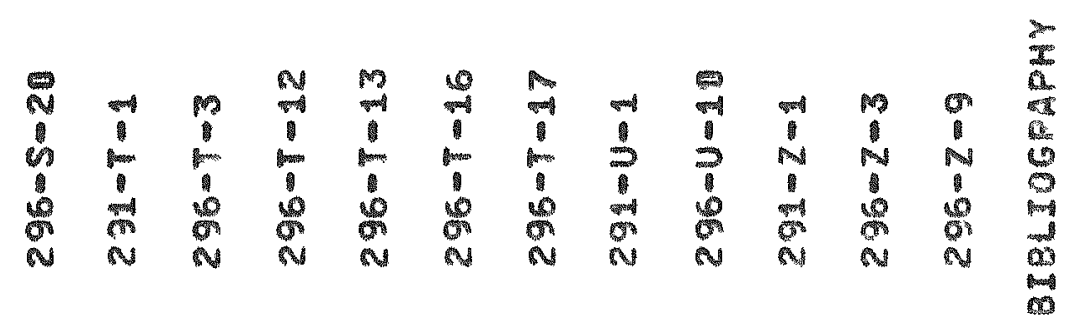




\section{INTRODUCTION}

This document is issued quarterly for the purpose of summarizing the radioactive gaseous wastes that are discharged from facilities of the Production and Waste Management Division, Atlantic Richfield Hanford Company. Data on alpha and beta emissions during 1975 are presented where relevant to the gaseous effluent. Emission data are not included on gaseous wastes produced within the 200 Areas by other Hanford contractors.

\section{SUMMARY}

During 1975, approximately $1.33 \times 10^{13}$ 1iters of gaseous effluents were monitored for radioactivity by the stack sampling program. A summary of the measured radioactivity was as follows:
A7pha (assumed source - Pu)
$1.45 \times 10^{-3}$ curie
Beta
0.418 curie

\section{DISCUSSION}

The volume and activity estimates of the gaseous effluents are detailed on a month-to-month basis for each site. The average activity, maximum activity, and the average concentration 
discharged from each stack are also presented for each site. Identification of stacks was based upon Item 2 of the Bibliography which used 291- as the prefix for 200-foot main stacks and 296- as the prefix for other stacks. Also listed in this report is a summary table which contains a listing of the activity for the current year, from startup and decayed from startup. Discharges from one new stack (296-T-17) are reported.

Gaseous effluents from Production and Waste Management Division stacks are analyzed for alpha and beta activity. The Purex process and the AR Vault waste facilities were not monitored for ${ }^{131}$ I emission because fuels processing during the report period was suspended. Analyses for other radioisotopes were performed where warranted by the alpha or beta activity level.

The data presented in the tables are to be read as shown by the following examples:

$$
\begin{aligned}
.241 E+07 & =0.241 \times 10^{+7} \\
.241 E-07 & =0.241 \times 10^{-7}
\end{aligned}
$$

The data in this report supersede that contained in the first three quarters' report (ARH-CD-370 30). 


\section{SUMMAPY OF RADIOACTIVE GAS OISCHARGED TO THF ATMOSPHERF}

200 FAST

FPOI $1 / 1$ TO $12 / 301975$

$\begin{array}{ll}\text { VOLUMA } & L \\ y & C I \\ 3=-2 & C I \\ 1131 & C I\end{array}$

THPNUGH $12 / 301975$ (FPOF STARTUPI

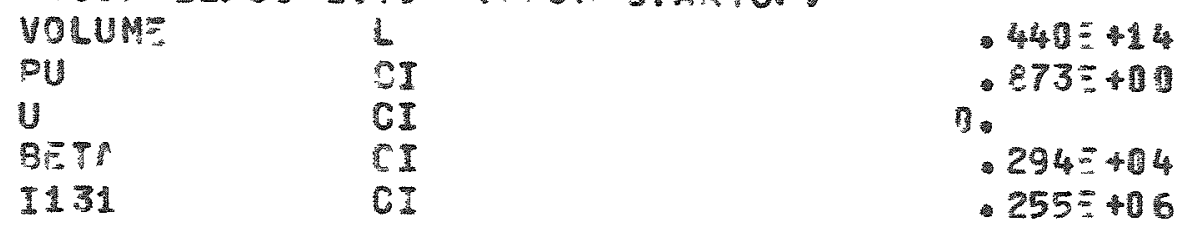

OFCAYO THROUGH 127301975 FPOH STARTUE
$3=7$
CI
$286=42$
131
CI

200 WEST

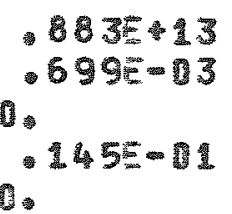

D.

695514

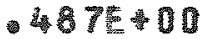

$-354 E-01$

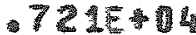

.219 .06

281E- 2
TOTAL

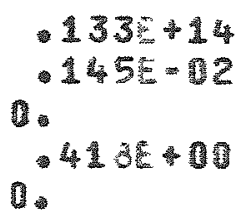

-114 45

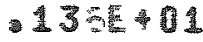
- 35 4 - 102 . $-475 E+06$ 


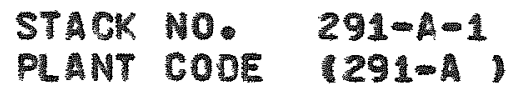

FACILITY PUREX

FUNCTION AIR FROM 291-A FILTERS. INCLUOES CANYON VENTILATION AR, VESSEL, AND CONDENSER VENTS. TREATED OISSOLVER OFF GAS.
$296-A-1$

\section{PUREX}

FILTERED AIR FROM N CELLS AMD ROR ROOM.

\begin{tabular}{|c|c|c|c|c|c|c|c|}
\hline $\begin{array}{l}\text { JAN } \\
\text { FP } 9 \\
\text { MAR } \\
\text { APR } \\
\text { MAY } \\
\text { JUH } \\
\text { WU } \\
\text { AUG } \\
\text { SEP } \\
\text { OOT } \\
\text { NOU } \\
\text { BEC } \\
\text { ADU }\end{array}$ & 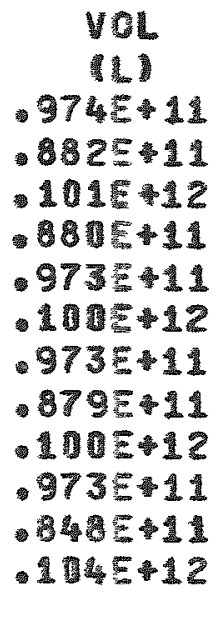 & $\begin{array}{c}P U \\
1 C I \\
280 E-04 \\
135 E-14 \\
177 E-04 \\
136 E-04 \\
908 E-05 \\
675 E=04 \\
392 E-04 \\
125 E-04 \\
.981 E-14 \\
223 E-04 \\
.42 E-14 \\
709 E-04\end{array}$ & 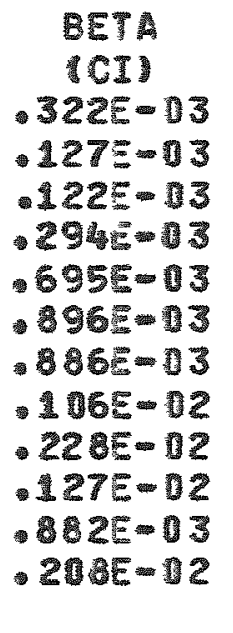 & $\begin{array}{l}T-131 \\
(C I)\end{array}$ & $\begin{array}{c}\text { VOL } \\
\text { IL } \\
.565 E+10 \\
551 E-10 \\
563 E+10 \\
7045.10 \\
564 E+10 \\
703 E+10 \\
583 E+10 \\
.563 E+10 \\
704 E-10 \\
586 E+10 \\
533 E+10 \\
704 E-10\end{array}$ & 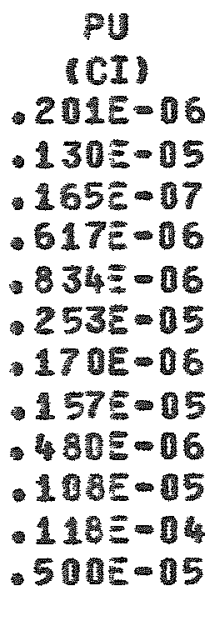 & 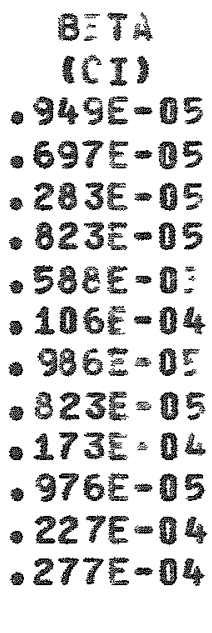 \\
\hline 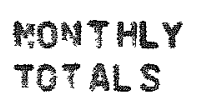 & $114 E+13$ & $-438 E-63$ & $-109 z-11$ & & $.733 E 11$ & $.256 \equiv-04$ & $.140 E-03$ \\
\hline $\begin{array}{l}\text { MONTHEY } \\
\text { MEXIMUN } \\
\text { FELCOSE }\end{array}$ & & $\begin{array}{l}\text { ICIVCA } \\
306 E-15\end{array}$ & $\begin{array}{l}\text { CI/OAY } \\
713=-14\end{array}$ & OCIODAY & & 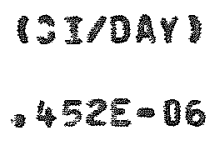 & $\begin{array}{l}\text { RCE/OAY } \\
.871 E=06\end{array}$ \\
\hline $\begin{array}{l}\text { AVERAGE } \\
\text { PELESSE }\end{array}$ & & $.121 E-65$ & 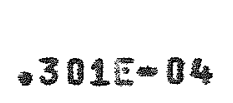 & & & $.593:=07$ & $.378 E=06$ \\
\hline 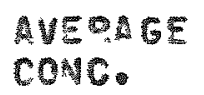 & & $\begin{array}{l}\text { UCI ICC } \\
-33 E-12\end{array}$ & $\begin{array}{r}\text { UCI/CC } \\
954=-11\end{array}$ & TUCIOCO & & $\begin{array}{l}\text { UCI/CSI } \\
349=-12\end{array}$ & $\begin{array}{l}\text { UCI/CCI } \\
190=11\end{array}$ \\
\hline
\end{tabular}


STMCK NO. 296-M-2

PLAN COOE $T-2$

\section{FACILITY PUPE}

FUNCTION HOOO EXHAUST FROM WEST SMDL GHLE:

\begin{tabular}{|c|c|c|c|}
\hline 1975 & $\begin{array}{l}106 \\
15\end{array}$ & $\begin{array}{l}\text { PU } \\
1621\end{array}$ & 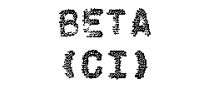 \\
\hline 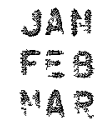 & $\begin{array}{l}311510 \\
299=-10 \\
-301510\end{array}$ & $\begin{array}{r}912=06 \\
1.125-17 \\
.1472-15\end{array}$ & 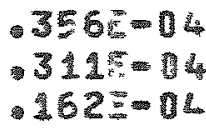 \\
\hline 政 & 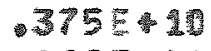 & $-2621-15$ & $-45=-4$ \\
\hline 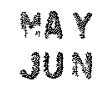 & $\begin{array}{l}299=110 \\
310=10\end{array}$ & $\begin{array}{l}.55 E-16 \\
.135 E=15\end{array}$ & $\begin{array}{l}.595-04 \\
7545-14\end{array}$ \\
\hline 10 & $\begin{array}{l}374=1 \\
-299=10\end{array}$ & $\begin{array}{l}2735-05 \\
-1505-05\end{array}$ & 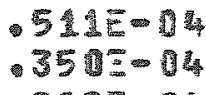 \\
\hline$S=$ & $-379=41$ & $231=6$ & $-69=013$ \\
\hline $93=$ & $-320=10$ & $-491-16$ & $-43=04$ \\
\hline MOY & 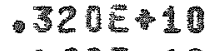 & $154=05$ & $-350=0$ \\
\hline $\mathrm{Cs}$ & $-90=19$ & $-325=5$ & $116=-3$ \\
\hline
\end{tabular}

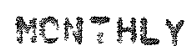

\begin{tabular}{|c|c|c|c|}
\hline TOTAS & $-396=11$ & .575014 & $.617=-13$ \\
\hline $\begin{array}{l}\text { MONTHLY } \\
\text { MXISUM }\end{array}$ & & 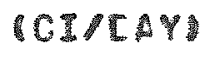 & 1CT/R离翼 \\
\hline$x_{-1}=$ & & $-932 E-17$ & $-3325-15$ \\
\hline 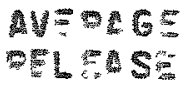 & & $-425 E-17$ & $-67 E=05$ \\
\hline 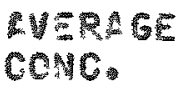 & & $\begin{array}{l}\text { UUI/CC } \\
-397=12\end{array}$ & 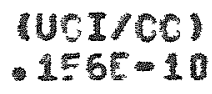 \\
\hline
\end{tabular}

\section{$2 y 6-3$

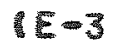

\section{PU}

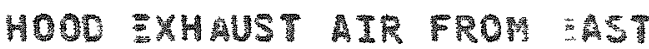

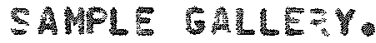

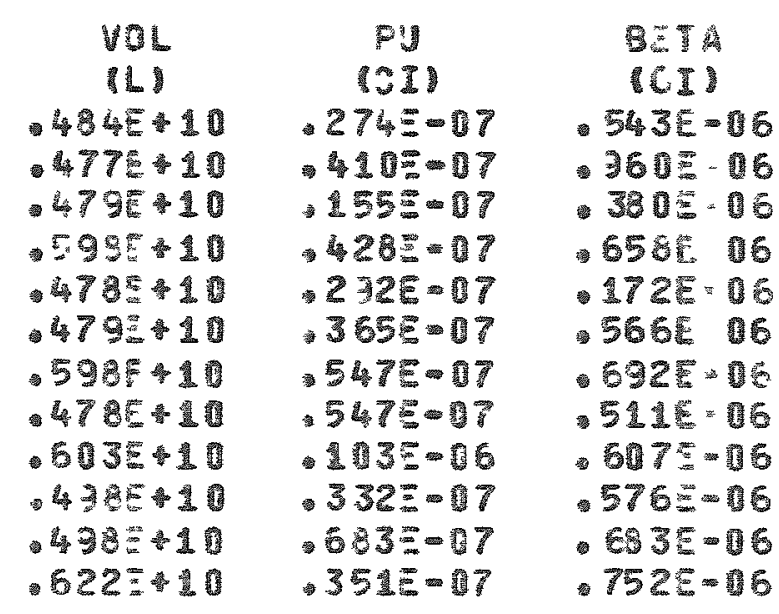

\begin{tabular}{|c|c|c|}
\hline \multirow[t]{5}{*}{$527=12$} & $5+15=6$ & $.7105-05$ \\
\hline & 431041 & I \\
\hline & $235=08$ & 343507 \\
\hline & 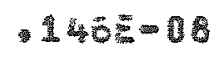 & .9207 \\
\hline & 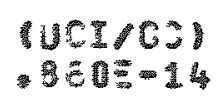 & 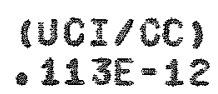 \\
\hline
\end{tabular}




\begin{tabular}{|c|c|}
\hline $\begin{array}{l}\text { STAON NO. } \\
\text { FLANAT COOE }\end{array}$ & $\begin{array}{l}296-4-5 \\
1 E-5-4\end{array}$ \\
\hline FACILIT & PUREX \\
\hline FUNG ION & 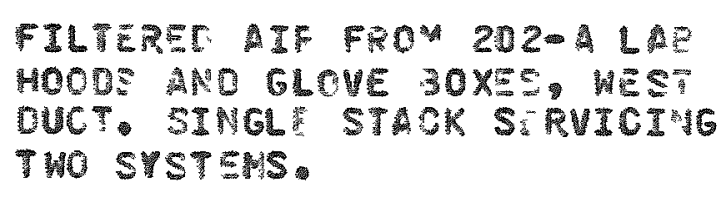 \\
\hline
\end{tabular}

$29-4-6$
$2-6$

pบ $\operatorname{cec}$

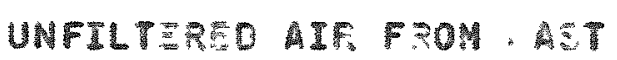
SAMPLE GALLESY MAO U CULL.

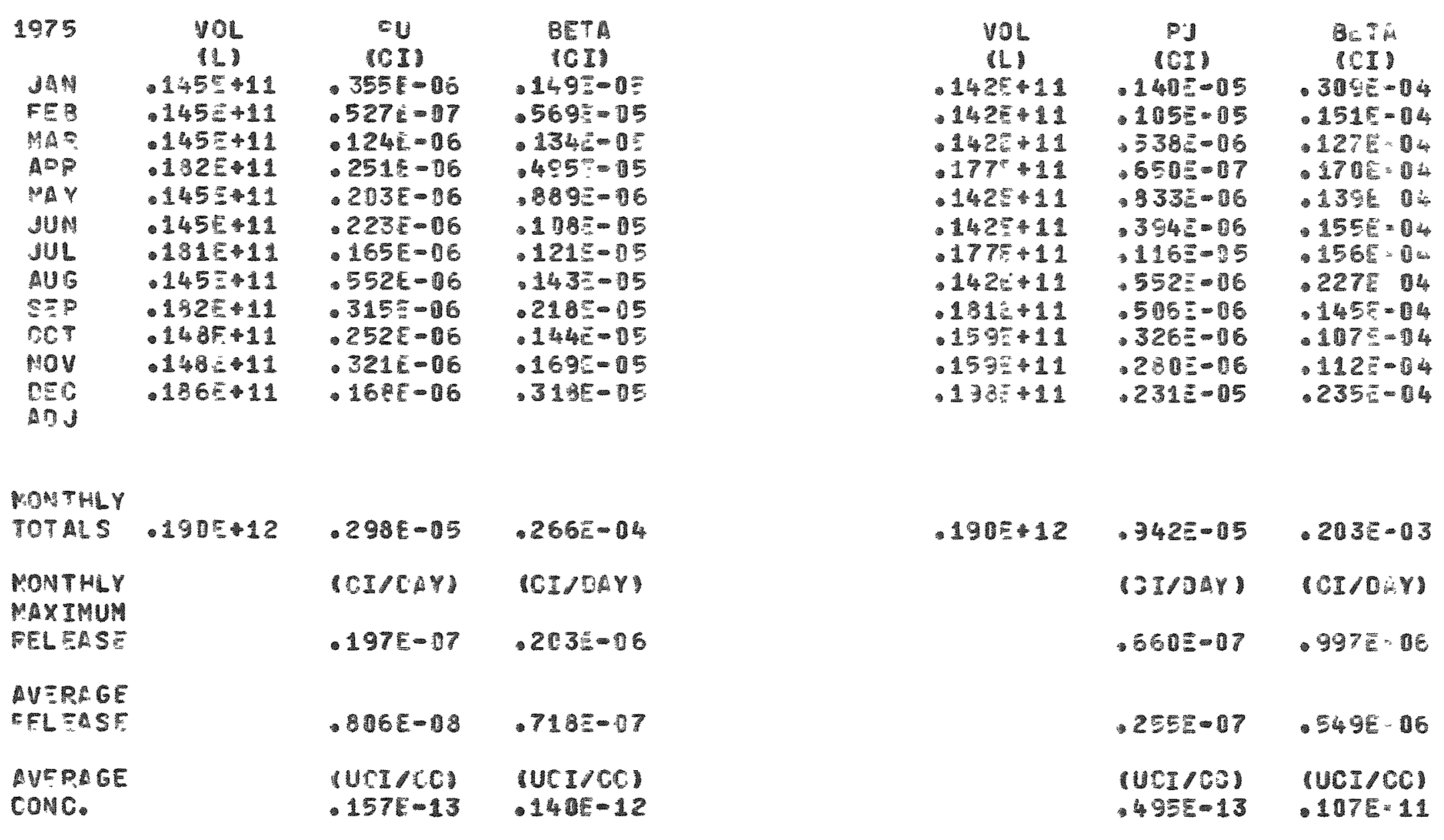


STACK NO. 296-A $=7$

PLANT CODE IE-?

\section{FACILITT PUREX}

FUNCIION UNFILTERED WIR FRON WEST SAMPLE GALEEPY AND R CELL.

\begin{tabular}{|c|c|c|c|}
\hline 1975 & VOL & PU & BEE \\
\hline $\begin{array}{l}\text { JAM } \\
F=8\end{array}$ & $\begin{array}{c}\text { ILI } \\
207 \%=1 \\
206=11\end{array}$ & $\begin{array}{c}\text { ICI } \\
.531 E=05 \\
.171 E=04\end{array}$ & $\begin{array}{c}1 C I \\
.578=-03 \\
144=02\end{array}$ \\
\hline 49 & 206611 & $158-15$ & $-169=3$ \\
\hline 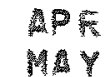 & $\begin{array}{l}2577^{*} \cdot 11 \\
205 \\
25 \cdot 11\end{array}$ & $\begin{array}{l}-522-05 \\
-326=05\end{array}$ & $\begin{array}{l}470=03 \\
199=03\end{array}$ \\
\hline MUn & $206 \% 11$ & $-875=06$ & $-7650=04$ \\
\hline $\begin{array}{l}\text { आUL } \\
\text { AUG }\end{array}$ & $\begin{array}{l}2575+11 \\
206 E+11\end{array}$ & $\begin{array}{l}171 E-05 \\
151 E-05\end{array}$ & $\begin{array}{l}1425-13 \\
-956=-04\end{array}$ \\
\hline SE\% & $259: 11$ & $.465 E=05$ & $.658 E=04$ \\
\hline OCT & $2165+11$ & $-237 E-05$ & $1415-03$ \\
\hline NOY & $-216 \div+11$ & $150 E-05$ & $1794=-04$ \\
\hline $0: c$ & $270=11$ & - 349E=05 & $1705-03$ \\
\hline
\end{tabular}

MCNTHLY

\begin{tabular}{|c|c|c|c|}
\hline TOTALS & $271 \leq 12$ & - $497 E-14$ & $.3635-12$ \\
\hline $\begin{array}{l}\text { MONTHLY } \\
\text { MXIYUN }\end{array}$ & & SCICAVI & ICINOA V \\
\hline FELEASF & & $.611 E-06$ & $-5145-74$ \\
\hline $\begin{array}{l}\text { METEGE } \\
\text { PELELSE }\end{array}$ & & .134506 & $9805-15$ \\
\hline $\begin{array}{l}\text { AVEPAGE } \\
\text { CONG. }\end{array}$ & & $\begin{array}{l}\text { RUCI/CCI } \\
-163 F=12\end{array}$ & $\begin{array}{l}\text { UCI/CC } \\
-1345-10\end{array}$ \\
\hline
\end{tabular}

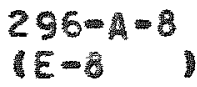

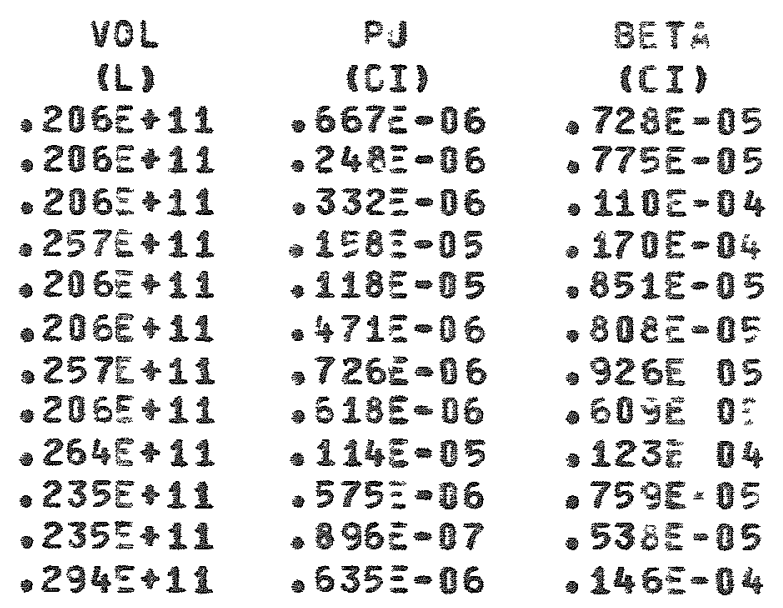

\begin{tabular}{|c|c|c|}
\hline \multirow[t]{5}{*}{2735} & $.826 E-05$ & $.115 \bar{E}-03$ \\
\hline & ISIDAY & ICI/OAY \\
\hline & $-51 \equiv-07$ & $-486 \cdot \sqrt{6}$ \\
\hline & $223 E-07$ & .310506 \\
\hline & $\begin{array}{l}\text { 105T/C31 } \\
297=-13\end{array}$ & $\begin{array}{l}\text { UCI/CC } \\
-413 E-12\end{array}$ \\
\hline
\end{tabular}




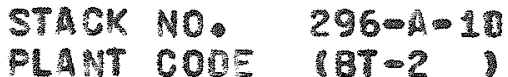

FACIITH PUPEX

FUNG I I ON FITERED ATE FROM BUFIAL TUNHEL NO. 2 .

\section{$296-12$ \\ (A⿵U}

244-R VMULT

FILTERED ATR FROM VESSEL VENH SYSTEW.

\begin{tabular}{|c|c|c|}
\hline 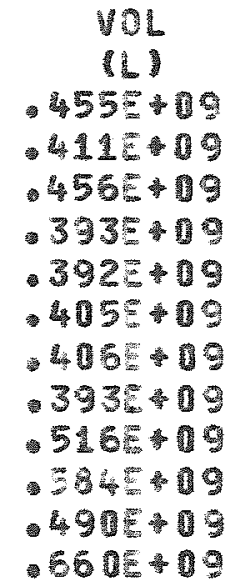 & 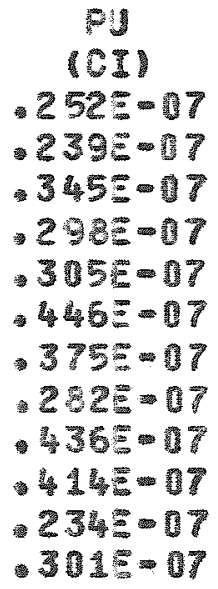 & 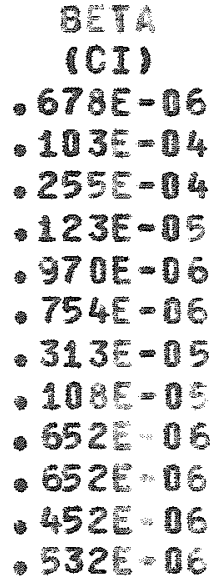 \\
\hline
\end{tabular}

\begin{tabular}{|c|c|c|c|}
\hline \multirow[t]{5}{*}{ 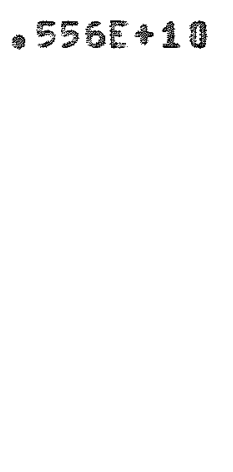 } & $.3938-06$ & -4595.42 & \\
\hline & 102/04Y & OCIOAY) & $13 I / 04 \%$ \\
\hline & $-1445=98$ & $.8235-16$ & \\
\hline & - $1135-68$ & $-1265-16$ & \\
\hline & $\begin{array}{l}\text { JUCE } 7001 \\
706-13\end{array}$ & $\begin{array}{l}\text { (UCI/CC) } \\
.8265-11\end{array}$ & $(001 / 60)$ \\
\hline
\end{tabular}

$I-131$ $(0 \pi)$
$.8265-11$ $-32=06$ - $243 E-06$ $-118 E-06$ $2465=06$ $107=06$ $-520=06$ $-212=16$ $-7007$ $.338=-16$

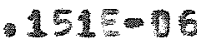
$.1945-16$ - $3445=06$

$.2985=05$
ICI IOA

$.1865-07$

$.006=08$

VUCI/CC UCI/CC

$-110 E-13 \quad .642 E-13$

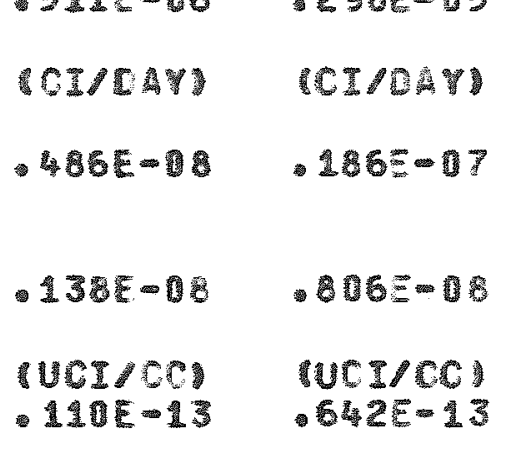


STACK NO. 296- 13

PLANT CODE PARCS

FACILITY 24M-AR VAULT

FUNCTION FILTERED AIF FROI GANYON AND CELL VENTILATION.

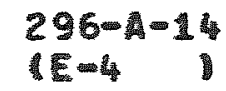

PUREX

FILTERE AIR FROM 293-A BUILDING.

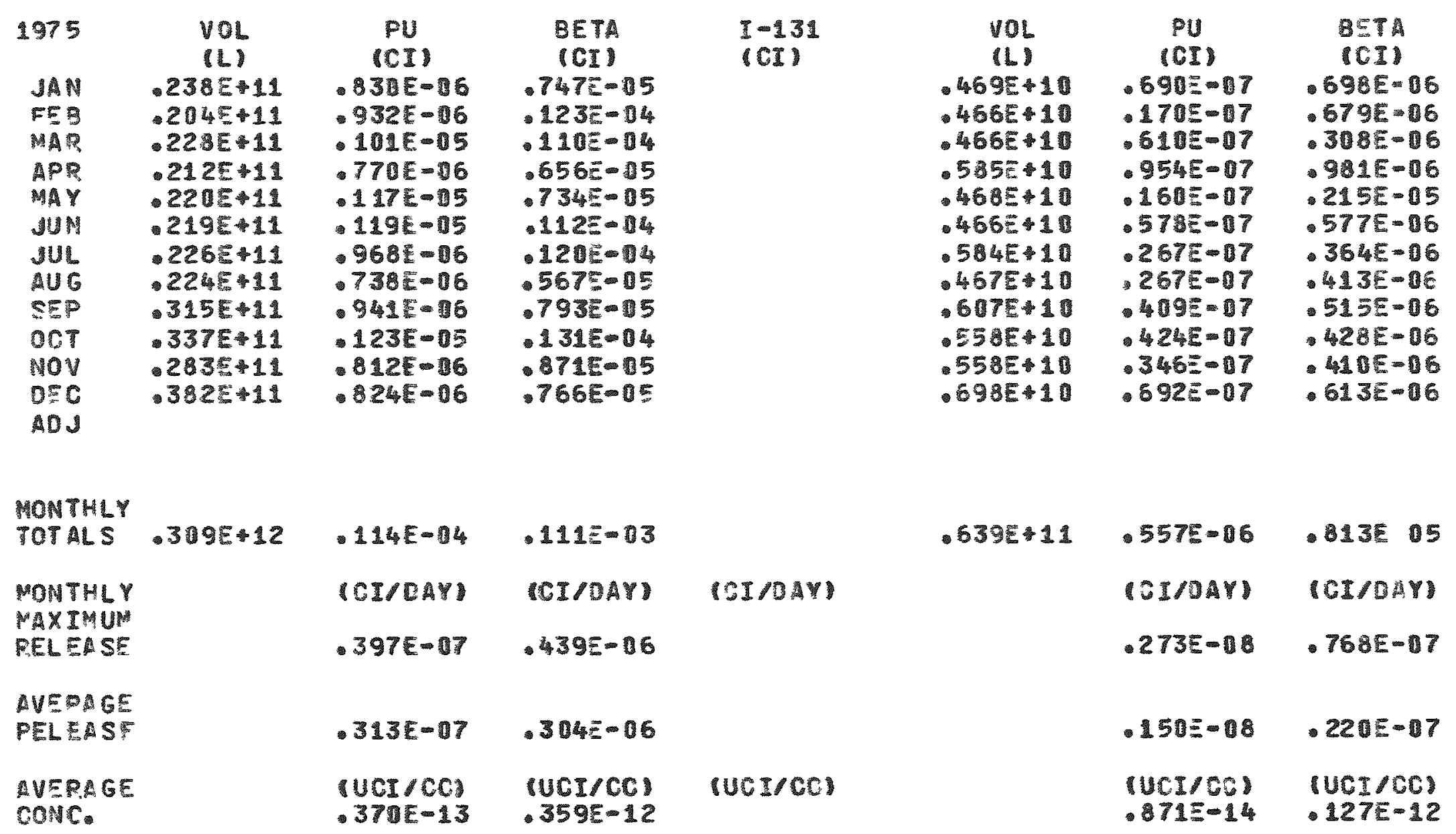


STACK NO. $296-17$
PLANT COOE IAXF

FACILITY 241A.AX,AY TANK FARM

FUNCTION FILTEREC NONCONOENSIBLE VAPORS FROM WASTE STORAGE TANKS IN 241-A. AX ANO AY TANK FARMS.

\section{$296-A-18$}

1101-AY

\section{1-AY TMUK ANHULUS}

FTLTERED VENT 241-AY-IOI TH MNULUS.

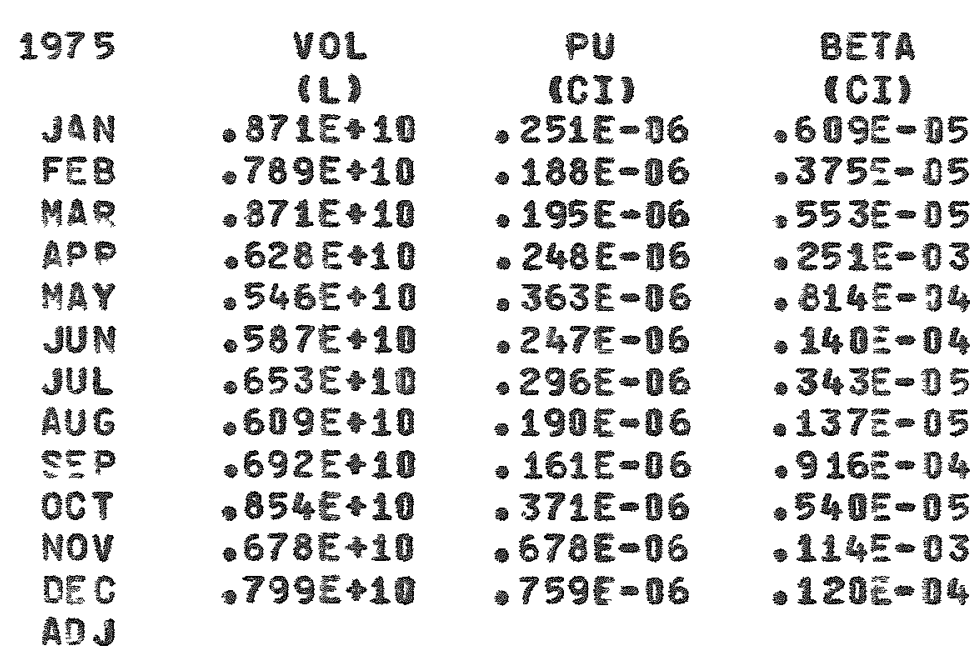

\begin{tabular}{|c|c|c|}
\hline 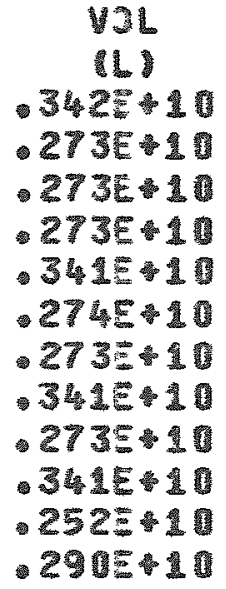 & 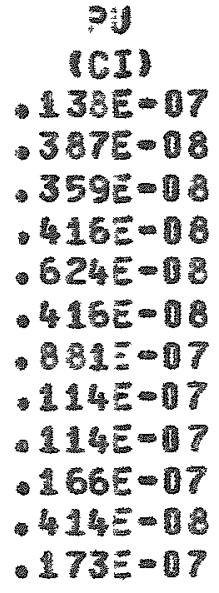 & 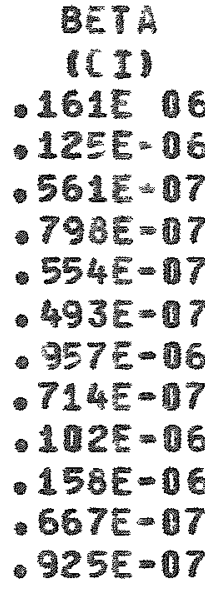 \\
\hline
\end{tabular}

MONTHLY

\begin{tabular}{|c|c|c|c|}
\hline TOTHS & 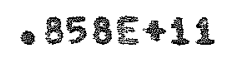 & $-395=05$ & $.590-03$ \\
\hline MON THEY & & ICI/DAY & ICI/D毒Y \\
\hline MAX IMUM & & & \\
\hline RFLEASE & & $-271 E=07$ & $.3372-05$ \\
\hline $\begin{array}{l}\text { AVERAGE } \\
\text { PELELSE }\end{array}$ & & - 109E-07 & $-162 E-15$ \\
\hline $\begin{array}{l}\text { AVERAGE } \\
\text { CONC. }\end{array}$ & & $\begin{array}{l}\text { UCI/CCI } \\
-460-13\end{array}$ & $\begin{array}{l}\text { UCI/CC) } \\
.68 B=11\end{array}$ \\
\hline
\end{tabular}

\begin{tabular}{|c|c|c|}
\hline \multirow[t]{5}{*}{. 355511} & $185=-16$ & $197 \mathrm{E}-0 \bar{z}$ \\
\hline & TCIOAYI & (CI/DAY) \\
\hline & $.315 E-16$ & $-342 E=07$ \\
\hline & $.509=09$ & - 54 \\
\hline & $\begin{array}{l}\text { 1 JCI/CG } \\
.521 \mathrm{E}-14\end{array}$ & $\begin{array}{l}\text { UCE } / C C 1 \\
.557 E-13\end{array}$ \\
\hline
\end{tabular}


STACY NO. $\quad 296-A-19$

PLANT CODE (102-AYI

FACILITY 102-AY TANK ANNULUS
FUNCTION FILTERED VENT $241-A Y-102$ TFNK
ANNULUS.

$291-B=1$ $(291-B)$

8 PLANT 1221-8)

ATR FROM 291-8-1 FILTE T. INCLUDES CANYON VEMTILATION ANO AII FOOH THE NO 1 PFOCESS VESSEL VENT.

\begin{tabular}{|c|c|c|c|c|c|c|}
\hline 1975 & VOL & PU & $\begin{array}{l}\text { BET } \\
\text { (CII }\end{array}$ & VOL & 10I & $\begin{array}{l}\text { BET } \\
\text { COI }\end{array}$ \\
\hline $\operatorname{MN}$ & $.447 E-10$ & - $4105-78$ & $.402 E=07$ & $.547 E-11$ & $.781 E=65$ & $.7998=02$ \\
\hline $\begin{array}{l}\text { FEB } \\
\text { MED }\end{array}$ & $\begin{array}{l}-358 E+10 \\
-357 E+10\end{array}$ & $\begin{array}{l}-382 E-B 8 \\
-382 E-18\end{array}$ & $\begin{array}{l}.735=-07 \\
.89 E=07\end{array}$ & $\begin{array}{l}.4945-11 \\
.5475-11\end{array}$ & $\begin{array}{l}9095-05 \\
100=04\end{array}$ & $\begin{array}{l}.322 E=01 \\
.54=01\end{array}$ \\
\hline MOF & 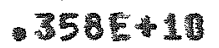 & $-109 E-37$ & - $74.5-17$ & $.535=11$ & $.405 E=05$ & $-170 E-01$ \\
\hline 新骨算 & $447=10$ & $136=07$ & $.775=07$ & $.550=11$ & $.927 E-05$ & - $418 E=02$ \\
\hline IUN & $-358 E+10$ & - 191E-67 & -746E-07 & $.5427-11$ & $-513 E-05$ & - $348 E=1$ \\
\hline JUL & $.357 E+10$ & $.629 \varepsilon-07$ & $-6875-16$ & $.555 \%+11$ & $1298=04$ & $-362 E=01$ \\
\hline AUG & 14 $45+10$ & $-275 E-67$ & $1465-16$ & $516 \%+11$ & $-527 E=05$ & 17482 \\
\hline $\begin{array}{l}\text { SEP } \\
0,7\end{array}$ & $\begin{array}{l}.357 E+10 \\
.447 E+10\end{array}$ & $\begin{array}{l}245 E-07 \\
.286 E-97\end{array}$ & $\begin{array}{l}954 E-07 \\
184 E-06\end{array}$ & $\begin{array}{l}567=11 \\
531+1\end{array}$ & $\begin{array}{l}143 E=04 \\
149 E=04\end{array}$ & $\begin{array}{r}981 E .01 \\
372 E .01\end{array}$ \\
\hline nov & -37? & $-230 E-17$ & - 184E- 16 & $.508=11$ & $.712 \xi=05$ & $.279-01$ \\
\hline $\begin{array}{l}\text { DEC } \\
\text { ADS }\end{array}$ & $.313 E+10$ & $.952 E-08$ & $.929 E-07$ & $-526=11$ & $.971 E=05$ & .277501 \\
\hline \multicolumn{7}{|l|}{ MONTHLY } \\
\hline TOTALS & $455 E 1^{1}$ & $.231 E-06$ & $-198 E-05$ & .6425 .12 & $.111 E-03$ & $.3852 \cdot 00$ \\
\hline NONTHLY & & (CI/OAY) & COIDAY & & (SIOAAY) & (CT/OAV) \\
\hline \multicolumn{7}{|l|}{ 鹖 } \\
\hline ELEASE & & - $225=08$ & $-317=07$ & & $-21 E-16$ & $-316 E-02$ \\
\hline \multicolumn{7}{|l|}{ AVERAGE } \\
\hline DELSASE & & $.637 c-09$ & $544 E-0$ & & $-306 z-16$ & $-106 E-02$ \\
\hline AVERAGE & & UCI/CCl & UCI I CC & & UCI/CE & UGI/CCI \\
\hline Conc. & & - $5799-14$ & $.43 E-13$ & & $-172 E=12$ & $.600 E=09$ \\
\hline
\end{tabular}




\begin{tabular}{|c|c|}
\hline $\begin{array}{l}\text { STACK NO: } \\
\text { PLNNE CODF }\end{array}$ & $\begin{array}{l}296-8-2 \\
|101-8|\end{array}$ \\
\hline FACILITY & ITS NO 1 \\
\hline FUNGTION & $\begin{array}{l}\text { FILTERED OFE GAS FROM THE } \\
\text { PROGESS BXHAUST TRMN. }\end{array}$ \\
\hline
\end{tabular}

\begin{tabular}{|c|c|c|c|}
\hline 1975 & $\begin{array}{l}\text { 10L } \\
\text { PL }\end{array}$ & $\begin{array}{c}\text { PU } \\
\text { CII }\end{array}$ & $\begin{array}{l}\text { BET } \\
\text { COI }\end{array}$ \\
\hline 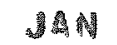 & $730=10$ & $.253 E-75$ & $241 x-7$ \\
\hline$F=8$ & $.6505 \cdot 10$ & $-136 E-155$ & $-167=-14$ \\
\hline A & 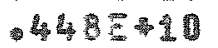 & $.515 E=06$ & $132=-14$ \\
\hline 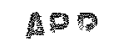 & $330 \pi+10$ & $-284 E=76$ & $1425-15$ \\
\hline 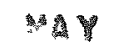 & .706510 & $-113 x-85$ & $.459=-104$ \\
\hline 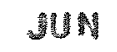 & .70758 & $.685 E-16$ & $-2765-04$ \\
\hline औU & $.7290^{\circ} \cdot 10$ & - $100-05$ & $-4595-14$ \\
\hline AUG & $682=-10$ & - $1418-05$ & $-9040-15$ \\
\hline$S \equiv D$ & $.753 E+10$ & $-433 E-06$ & $.505=05$ \\
\hline Cet & .729530 & $.665=06$ & $.579=-15$ \\
\hline Nov & .6125 .10 & - $10 E-06$ & $-445 E-15$ \\
\hline$s=0$ & - 323 E 10 & - 409E-06 & $.307 E-05$ \\
\hline and & & & \\
\hline
\end{tabular}

MONTHLY

\begin{tabular}{|c|c|c|c|}
\hline TOTALS & $.791 \vec{E}-11$ & $-108=04$ & $202=-3$ \\
\hline $\begin{array}{l}\text { MONTHEY } \\
\text { MYTEUM }\end{array}$ & & ICIACAV & GCI/OAY \\
\hline Fel cese & & - $3168-97$ & - $153=05$ \\
\hline $\begin{array}{l}\text { AVTREGE } \\
\text { RELESS: }\end{array}$ & & $3105-97$ & $.5785-06$ \\
\hline $\begin{array}{l}\text { AVIDEGE } \\
\text { CONC. }\end{array}$ & & $\begin{array}{l}\text { UCT /CCI } \\
-137 E-12\end{array}$ & $\begin{array}{l}. U C I / C C 1 \\
.2565-11\end{array}$ \\
\hline
\end{tabular}

\section{$296-5=3$ \\ (112-81)}

ITS NO. 2

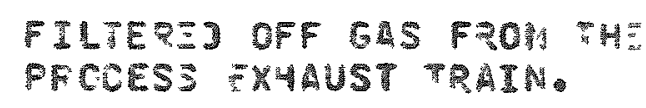

\begin{tabular}{|c|c|c|}
\hline $\begin{array}{c}\text { VL } \\
1 L \\
318=49 \\
203=-109 \\
310=09 \\
289=09\end{array}$ & $\begin{array}{c}\text { PU } \\
10 T \\
507=-17 \\
773=-17 \\
7719=-17 \\
.758=-07\end{array}$ & 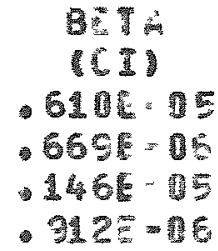 \\
\hline
\end{tabular}

\begin{tabular}{|c|c|c|}
\hline \multirow[t]{5}{*}{$-121 \leq 1$} & $.276=-16$ & $-914=05$ \\
\hline & 1CTOMPI & COIDCA \\
\hline & $-275-06$ & $1972=06$ \\
\hline & $232=00$ & .758 .07 \\
\hline & $\begin{array}{l}101 / 3=1 \\
2275=12\end{array}$ & 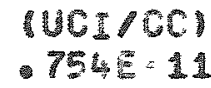 \\
\hline
\end{tabular}


STACK NO. 296-8-

PLANT CODE IS-4

\section{FHCILI霍 221-DB BUILOING}

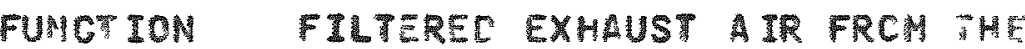

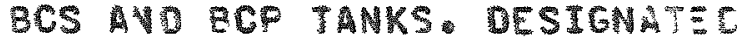
VSSEL VEMT MO

\section{$296-8=5$ \\ (221BBS)}

221-38 3UTLOING

DISCHA THE 221-6B BUILITG.

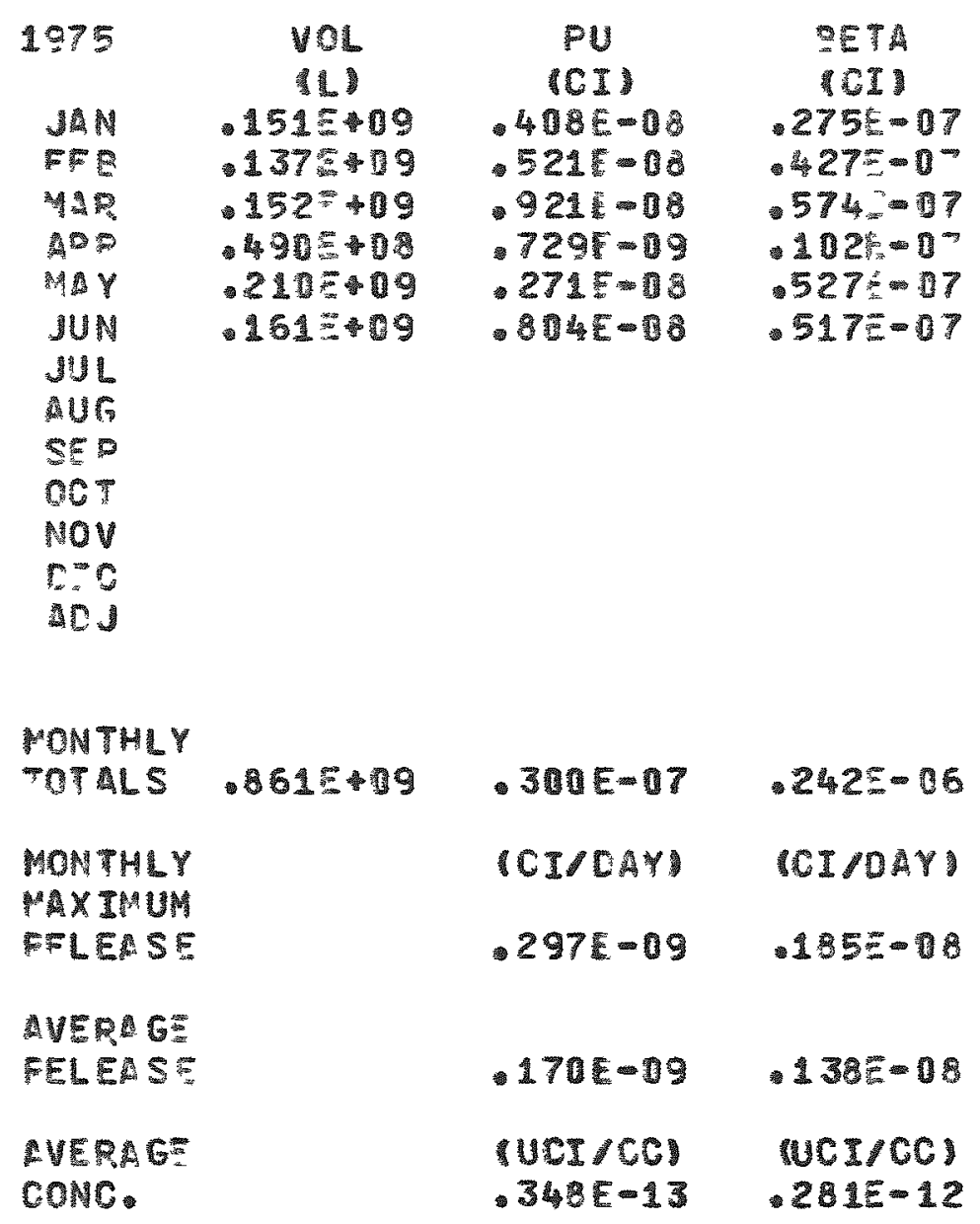

\begin{tabular}{|c|c|c|}
\hline \multirow[t]{5}{*}{.2638} & $1115=05$ & .186504 \\
\hline & (2I/OAY) & IOI/OAY \\
\hline & $-535=-0$ & $.163 E-06$ \\
\hline & $-305=0$ & - $512 E-07$ \\
\hline & 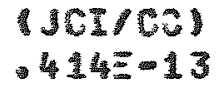 & $\begin{array}{l}\text { UCE/CCI } \\
.693 E-12\end{array}$ \\
\hline
\end{tabular}


STACH NO. 296-8-10

GLAN COOE I225B

FAILITY 225- SUILOTNG

FUNC ION FILTFE ED AIF FROM THE ENCAPSUL ATICN FACILITY.

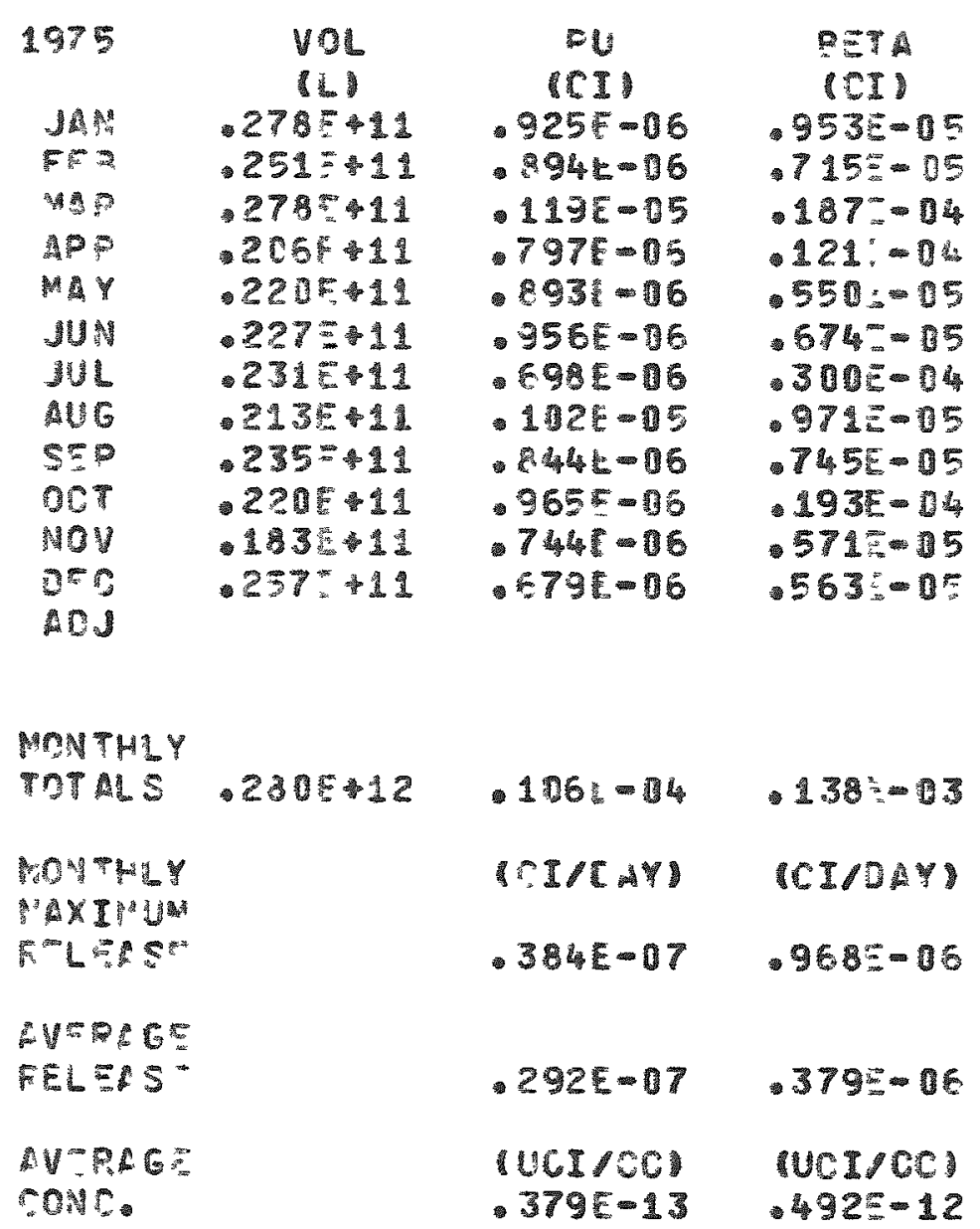

$291-C-1$
ISWS

\section{SEATHOKS (201-6)}

FILTFDO AR FPO' SELL VENTLLITCN ANO PSOC SE VESSEL VII.

\begin{tabular}{|c|c|c|}
\hline $\begin{array}{c}y=L \\
14 \\
131+11 \\
11+2=11\end{array}$ & $\begin{array}{c}P 1 \\
10 Z 1 \\
854=-16 \\
-43=-16\end{array}$ & $\begin{array}{c}3=1 \\
101 \\
-152=-10 \\
0825=05\end{array}$ \\
\hline $\begin{array}{l}130-11 \\
107-11 \\
107-107 \\
107-11\end{array}$ & $\begin{array}{r}131 z-05 \\
-17=06 \\
-336=06\end{array}$ & $\begin{array}{l}.2045=04 \\
.1025-04 \\
-808505\end{array}$ \\
\hline 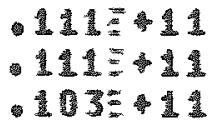 & $\begin{array}{l}232 E=05 \\
.230=-16 \\
.355 E-66\end{array}$ & $\begin{array}{l}-676 E \\
-706 E=04 \\
-32=-105\end{array}$ \\
\hline $\begin{array}{r}114 y^{*}+11 \\
-1115-11 \\
-923-10\end{array}$ & $\begin{array}{l}378=-15 \\
.475-06 \\
.403 E-06\end{array}$ & $\begin{array}{l}-237 E=64 \\
-3115=04 \\
.673 E-05\end{array}$ \\
\hline $12=11$ & $-430=-06$ & $-\rightarrow 38=05$ \\
\hline
\end{tabular}

\begin{tabular}{|c|c|c|}
\hline \multirow{5}{*}{$-133^{-1}-12$} & $.632=0.05$ & .263503 \\
\hline & PIDAY & $(C I / O-1)$ \\
\hline & $.423=-07$ & $.2705-05$ \\
\hline & $.173=-07$ & $.722-06$ \\
\hline & $\begin{array}{l}\text { UUCI/COI } \\
.464=-13\end{array}$ & $\begin{array}{l}\text { UCI/CG } \\
193=-11\end{array}$ \\
\hline
\end{tabular}


STACK NO. $\quad 295-\mathrm{C}-2$

PLANT CODE IWPS

\section{FACILITY SEMINOOKS $1201=0$ \\ FUNCTION FILTEREO AIF FFO A ANC C SAMPL GILEFES.}

\begin{tabular}{|c|c|c|c|}
\hline 1975 & Vol & PU & 9ETA \\
\hline & LI & (C) & (CII \\
\hline 或 & $144 y^{2}=10$ & $-2645=07$ & $-32 E-06$ \\
\hline f & $5975 \cdot 10$ & $189 E=07$ & $-169 e-06$ \\
\hline An & .595810 & . $2268=07$ & $-195=-16$ \\
\hline 100 & $-277=10$ & $.288 F=7$ & $.2885=06$ \\
\hline$M$ & $1347=10$ & & $400^{\circ}=0$ \\
\hline 约镜 & $-2768+11$ & $143 E-147$ & $6=06$ \\
\hline JUL & $.277 F^{2}-10$ & $-7041=0$ & $1=07$ \\
\hline MU & $137^{\circ} 10$ & $=-7$ & \\
\hline S:P & $-277^{2}+12$ & 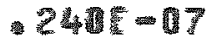 & $.7315=0$ \\
\hline $00 T$ & .3478 & - 352 $=7$ & $-111=06$ \\
\hline Mov & $.258=10$ & $-106 E-107$ & $-1045=06$ \\
\hline DEC & .2975 - 10 & $.704=8$ & $.335 E-06$ \\
\hline
\end{tabular}

MonTHLY

\begin{tabular}{|c|c|c|c|}
\hline TOTALS & 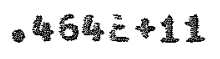 & $2{ }^{2} 15-06$ & $-214 E-15$ \\
\hline MONTHLY & & ICI/CAY & (CI/OAY) \\
\hline GXINUN & & & \\
\hline PLLESSE & & $-113 E-0 ?$ & $-1395-17$ \\
\hline MEPG & & & \\
\hline prickst & & $.663 E-09$ & $.5685=08$ \\
\hline $\begin{array}{l}\text { AVEDAGE } \\
\text { CONC. }\end{array}$ & & $\begin{array}{l}\text { VUCI/CCU } \\
.519 E-14\end{array}$ & $\begin{array}{l}\text { UCI/CC } \\
460 E-13\end{array}$ \\
\hline
\end{tabular}

\section{$296-c-5$ \\ CQV}

$244=C$ V VALT

FILTERE ATR FROM CELL VENTILATICN ANE PSOCXSS VESSEL VENT.

\begin{tabular}{|c|c|c|}
\hline VOL & $p U$ & 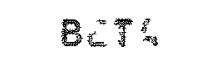 \\
\hline IL & IOI & (c) \\
\hline$B 3=10$ & $117 \bar{E}-15$ & $\begin{array}{r}111 E-14 \\
120 E-4\end{array}$ \\
\hline $\begin{array}{l}7 \text { घ. } 10 \\
03 E-10\end{array}$ & $\begin{array}{r}1125-05 \\
-1505-15\end{array}$ & $\begin{array}{l}120500 \\
1448\end{array}$ \\
\hline & $\begin{array}{r}180=-85 \\
.475-196\end{array}$ & $\begin{array}{l}175 E-14 \\
-765 E-15\end{array}$ \\
\hline 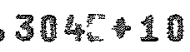 & $.1065=05$ & $-142=-4$ \\
\hline & $\begin{array}{r}-588=06 \\
365=-06\end{array}$ & $\begin{array}{r}3375=0 \\
3=0\end{array}$ \\
\hline 10 & $.2045-06$ & - $46 \mathrm{E} E=0$ \\
\hline & $-778=-16$ & - $177 E-1$ \\
\hline & $711=-16$ & $-120 E$ \\
\hline 10 & $.983 E-05$ & -138 \\
\hline
\end{tabular}

\begin{tabular}{|c|c|c|}
\hline \multirow[t]{5}{*}{ 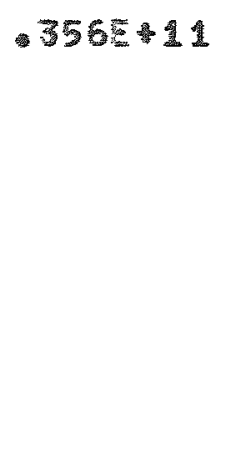 } & $-108=-04$ & $-137=-03$ \\
\hline & 101/3AY & |CI/DAY \\
\hline & $.6005-07$ & $-583 E-06$ \\
\hline & $.2975-07$ & $.376 E-06$ \\
\hline & $\begin{array}{l}\text { UCI/CCI } \\
304=-12\end{array}$ & $\begin{array}{l}\text { UCI/CC) } \\
.386 \hat{C}^{2} 11\end{array}$ \\
\hline
\end{tabular}




\begin{tabular}{|c|c|}
\hline $\begin{array}{l}\text { STACK NO. } \\
\text { PLANT CODE }\end{array}$ & $\begin{array}{l}296-1 \\
\ln =1\end{array}$ \\
\hline FACILITY & BX TANK FARE \\
\hline FUNG ION & $\begin{array}{l}\text { POPTABLE AIF EXHAUSTER FOF } \\
105.126,110 \text { AND } 111-9 x \\
\text { TANKS INBX FARM. }\end{array}$ \\
\hline
\end{tabular}

\begin{tabular}{|c|c|c|c|}
\hline \multirow[t]{2}{*}{1975} & yol & PU & BETA \\
\hline & 11 & 102 & CII \\
\hline JAN & $-4495-11$ & $.5445=06$ & $-3925=5$ \\
\hline$F \equiv B$ & -4650 & $2275-06$ & $-3105-05$ \\
\hline$M R$ & $-457=10$ & $-269-66$ & $.283 z-05$ \\
\hline 怘D & $-435 z+1$ & $-566-16$ & $-478=05$ \\
\hline HY & - $455+14$ & $.627=06$ & $-4295-15$ \\
\hline JUN & 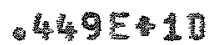 & $-255=06$ & $-32=-15$ \\
\hline JUL & $.450 E-10$ & $.282=06$ & $-46=05$ \\
\hline AUS & $.421=10$ & $.1585=06$ & $-2855=05$ \\
\hline SEO & $.4645+11$ & $.260 E-06$ & $-4215-05$ \\
\hline $00 \%$ & .450 .10 & $22^{2}-06$ & $.236 E-05$ \\
\hline NOV & . 377 C & $.2471=16$ & $391 E=05$ \\
\hline$n=0$ & 508510 & $.609 E-6$ & $-4265-05$ \\
\hline ADJ & & & \\
\hline
\end{tabular}

MONTHLY

\begin{tabular}{|c|c|c|c|}
\hline TETLE & .52951 & $.425 E=25$ & $-450 E-74$ \\
\hline MONTHLY & & ICI/DAY & (GI/OAY) \\
\hline MAX INUH & & & \\
\hline FLLEASE & & $.209 E-07$ & $1595-06$ \\
\hline $\begin{array}{l}\text { METGE } \\
\text { FELESSE }\end{array}$ & & $116 E-07$ & $-123 E-66$ \\
\hline $\begin{array}{l}\text { MPo } G= \\
\text { conco }\end{array}$ & & $\begin{array}{l}\text { UCI/CC) } \\
802 E-13\end{array}$ & $\begin{array}{l}\text { UUCI/CCI } \\
.849 E=12\end{array}$ \\
\hline
\end{tabular}

\section{$296-P-2$}

$1104-31$

\section{ITS NO. 2}

FILTESE EXHLUST ATR FrOM R COOLING THE CONCENTRA IN $\mathrm{BV}$

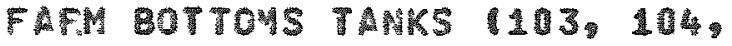
105. AND 105-8VI?

\begin{tabular}{|c|c|c|}
\hline 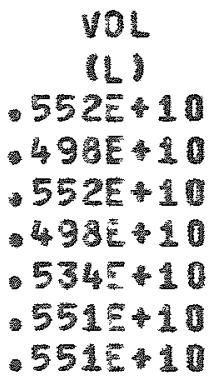 & 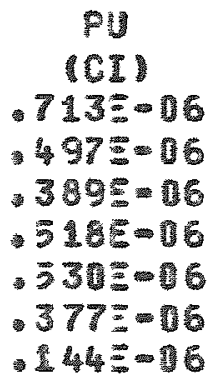 & 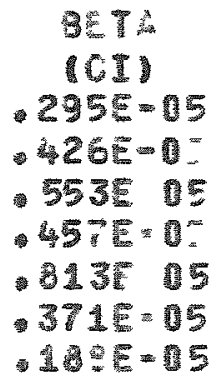 \\
\hline $\begin{array}{l}.516 E+10 \\
5695-10 \\
.551 E \cdot 10 \\
4425+10 \\
.671 E-10\end{array}$ & $\begin{array}{l}164=06 \\
.263=-16 \\
196=-06 \\
167=-06 \\
175=-06\end{array}$ & $\begin{array}{l}142 E-15 \\
.226 E=05 \\
.335=05 \\
-13 z=16 \\
.232 E=05\end{array}$ \\
\hline
\end{tabular}

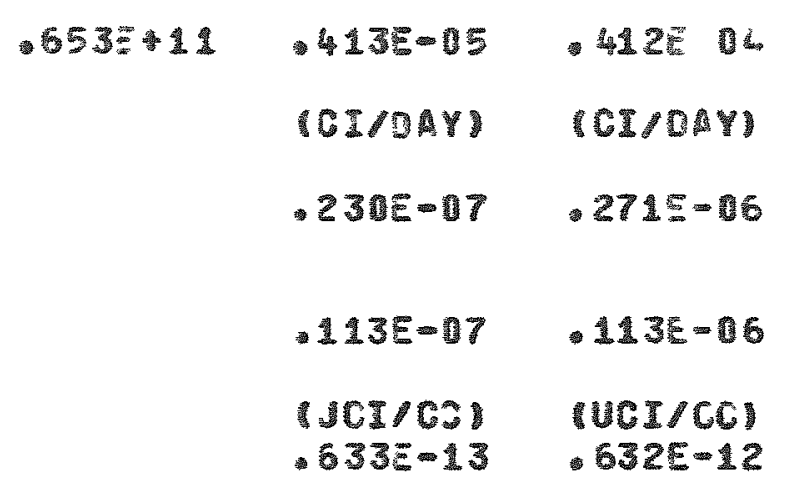


STCK $N O$. 295-P-3

PLANT CODE PINT-BY

FACIIIT ITS 10.2

FUMC TON FILTIRO EXMAUST AIR FRCH COCLING THE CONG NOPTE IN FY FAFM BOTTOMS TANKS 1107,128, 11), AND 111-BV?

\begin{tabular}{|c|c|c|c|}
\hline 1975 & $\begin{array}{l}\text { VOL } \\
\text { IL }\end{array}$ & $\begin{array}{c}\text { PU } \\
\text { 10I }\end{array}$ & $\begin{array}{l}\text { PETh } \\
\text { PCI }\end{array}$ \\
\hline JAN & $8918+10$ & $.1375=05$ & $891=-14$ \\
\hline$F E B$ & $.87 \%$ & $-152=05$ & $.509=04$ \\
\hline A & 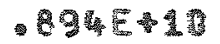 & $.6211=06$ & $146=03$ \\
\hline APR & .8625914 & $.151 z=75$ & $229=-02$ \\
\hline 期 & .8535 .14 & $-2275-06$ & $.576 E-14$ \\
\hline SUN & $.892=10$ & $-698 E-66$ & $-2635-12$ \\
\hline JUL & 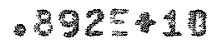 & $.562 e-76$ & $\left..25 \frac{4}{4}-1\right)$ \\
\hline 106 & .8358 .10 & $.6495-06$ & $.263 \equiv-04$ \\
\hline sep & $.921{ }^{5} \cdot 10$ & $3921=05$ & $.105 E=04$ \\
\hline ort & $-892 \div-10$ & $-116-25$ & $-1725-14$ \\
\hline Mov & $5868+10$ & $-245-06$ & $-1030^{2}=04$ \\
\hline DEC & $350 \div 10$ & $.874 x=7$ & $.239 E^{\circ}$ \\
\hline
\end{tabular}

$296-P=4$

$(109-3)$

ITS 10.2

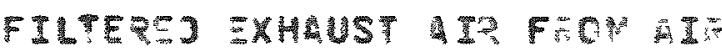
COOLING THE SONCENTRER. Th FAPA 3OTTOH TANK $109-5 Y 1$.

\begin{tabular}{|c|c|c|}
\hline 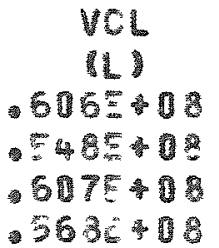 & $\begin{array}{c}F U \\
\text { CII } \\
450=08 \\
344=08 \\
302=08 \\
30=08 \\
216=08\end{array}$ & 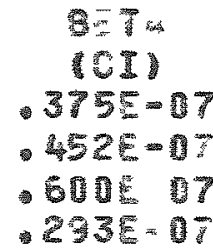 \\
\hline
\end{tabular}

MOHTHLY

\begin{tabular}{|c|c|c|c|}
\hline Tก A & $.969 E^{-11}$ & $964=05$ & $.507=-13$ \\
\hline $\begin{array}{l}\text { MONTHLY } \\
\text { MXI UN }\end{array}$ & & CCI/C & (CI/CAY) \\
\hline FELESE & & $.5435-07$ & $.471 E-0=$ \\
\hline $\begin{array}{l}\text { AVERAGE } \\
\text { CELEASE }\end{array}$ & & $264=07$ & $\cdot 1395-0$ \\
\hline $\begin{array}{l}\text { AVSRG } \\
\text { ronc. }\end{array}$ & & $\begin{array}{l}\text { UCI CCI } \\
996 \mathrm{E}-13\end{array}$ & UCI/CC \\
\hline
\end{tabular}

\begin{tabular}{|c|c|c|}
\hline \multirow[t]{5}{*}{ - 233: 09} & -1345: $=7$ & .1728 .06 \\
\hline & ICIOAY & (CI/OAY) \\
\hline & $-155 z-09$ & $-1345=0$ \\
\hline & $.113 \equiv=09$ & $.145=06$ \\
\hline & $\begin{array}{l}U C I / C O 1 \\
-5765-13\end{array}$ & $\begin{array}{l}\text { UCI/CC } \\
.738 E-12\end{array}$ \\
\hline
\end{tabular}




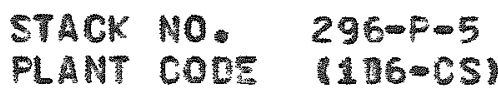

FACILITY TANKS 105 AND $106-\mathrm{C}$

FUNCTION PORTABLE AIT EXHAUSTIR FOR THE 195 AVD 106-C TANKS IN C FAS
$296-9-6$

1104

241-A TAMK FU RM

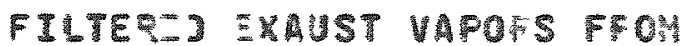

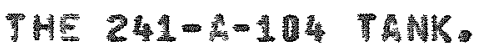

\begin{tabular}{|c|c|c|}
\hline $\begin{array}{l}\text { Vet } \\
\text { Pin }\end{array}$ & $\begin{array}{c}23 \\
1013\end{array}$ & 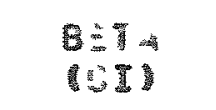 \\
\hline 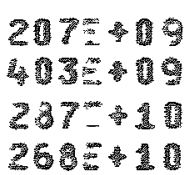 & $\begin{array}{r}.045=07 \\
.402=05 \\
.5110=06 \\
.1795-06\end{array}$ & $\begin{array}{l}7145-5 \\
.9545-15 \\
.535 E-15 \\
.221-05\end{array}$ \\
\hline $54=0$ & $346=08$ & $-127 E \cdot 05$ \\
\hline $325-10$ & $-180 z-15$ & $-191 \mathrm{E}=04$ \\
\hline & (OIDAY & 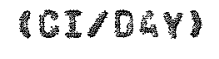 \\
\hline & - $329 \varepsilon-07$ & $127 \bar{c}=5$ \\
\hline & $-163 E-07$ & $.1745-06$ \\
\hline & $\begin{array}{l}\text { UCI/CSU } \\
-264 E-12\end{array}$ & $\begin{array}{l}\text { UCI/CC } \\
-302 E-11\end{array}$ \\
\hline
\end{tabular}

$26-12$

\begin{tabular}{|c|c|c|c|}
\hline $\begin{array}{l}\text { MONTHLY } \\
\text { TOTALS }\end{array}$ & -617E!1 & $-398=15$ & $.645=-04$ \\
\hline $\begin{array}{l}\text { MONTHLY } \\
\text { MX X MUUY }\end{array}$ & & TCI/DAV & (CI/OAY) \\
\hline TELEASE & & $-361 E-07$ & $-421 E-76$ \\
\hline 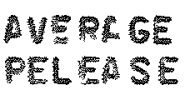 & & $109-07$ & $-1775-06$ \\
\hline $\begin{array}{l}\text { AVERGE } \\
\text { CONC. }\end{array}$ & & $\begin{array}{l}\text { UCI CCI } \\
.645 E=13\end{array}$ & $\begin{array}{l}\text { UCI/CC } \\
.105 E-11\end{array}$ \\
\hline
\end{tabular}

\begin{tabular}{|c|c|c|c|}
\hline $\begin{array}{l}\text { JAN } \\
F E B \\
\text { MAP }\end{array}$ & $\begin{array}{c}\text { VOL } \\
\text { TLI } \\
598 E 10 \\
.5418 .10 \\
.597 E .10\end{array}$ & $\begin{array}{c}P U \\
\text { CII } \\
592 E-106 \\
191 E=15 \\
716 E=06\end{array}$ & $\begin{array}{c}\text { BETM } \\
\text { CII } \\
592=-05 \\
.11=04 \\
536=05\end{array}$ \\
\hline Spo & 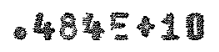 & $.293 E-06$ & $.538 E-15$ \\
\hline $\begin{array}{l}\text { MAY } \\
\text { WUN }\end{array}$ & 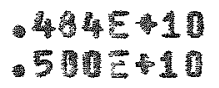 & $\begin{array}{l}-125 E-16 \\
.225 E-06\end{array}$ & $\begin{array}{r}1135=15 \\
394=-5\end{array}$ \\
\hline $\begin{array}{l}\text { SUL } \\
\text { AUE }\end{array}$ & 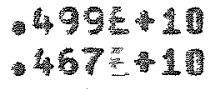 & $\begin{array}{l}13+15-16 \\
-136-06\end{array}$ & $\begin{array}{l}-3775-15 \\
-2165-15\end{array}$ \\
\hline$S=p$ & -515: & -112 管 $=06$ & $.6075-05$ \\
\hline 061 & $-499=10$ & $-2471-06$ & $-120=0$ \\
\hline Mov & $495-16$ & $224 E-16$ & $-2325=05$ \\
\hline DEC & 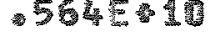 & $-276=-16$ & - $4695-05$ \\
\hline
\end{tabular}


$\begin{array}{ll}\text { STACK NO. } & 296-P-7 \\ \text { PLANT COOE } & 1098 \% 8\end{array}$

FACILITIVIS MI.

FUNOTION FILTERD EXHAUST AIR FFON AO COOLING THE CONCENTRATRE IA BY FARY SOTTOH TANK IISEY

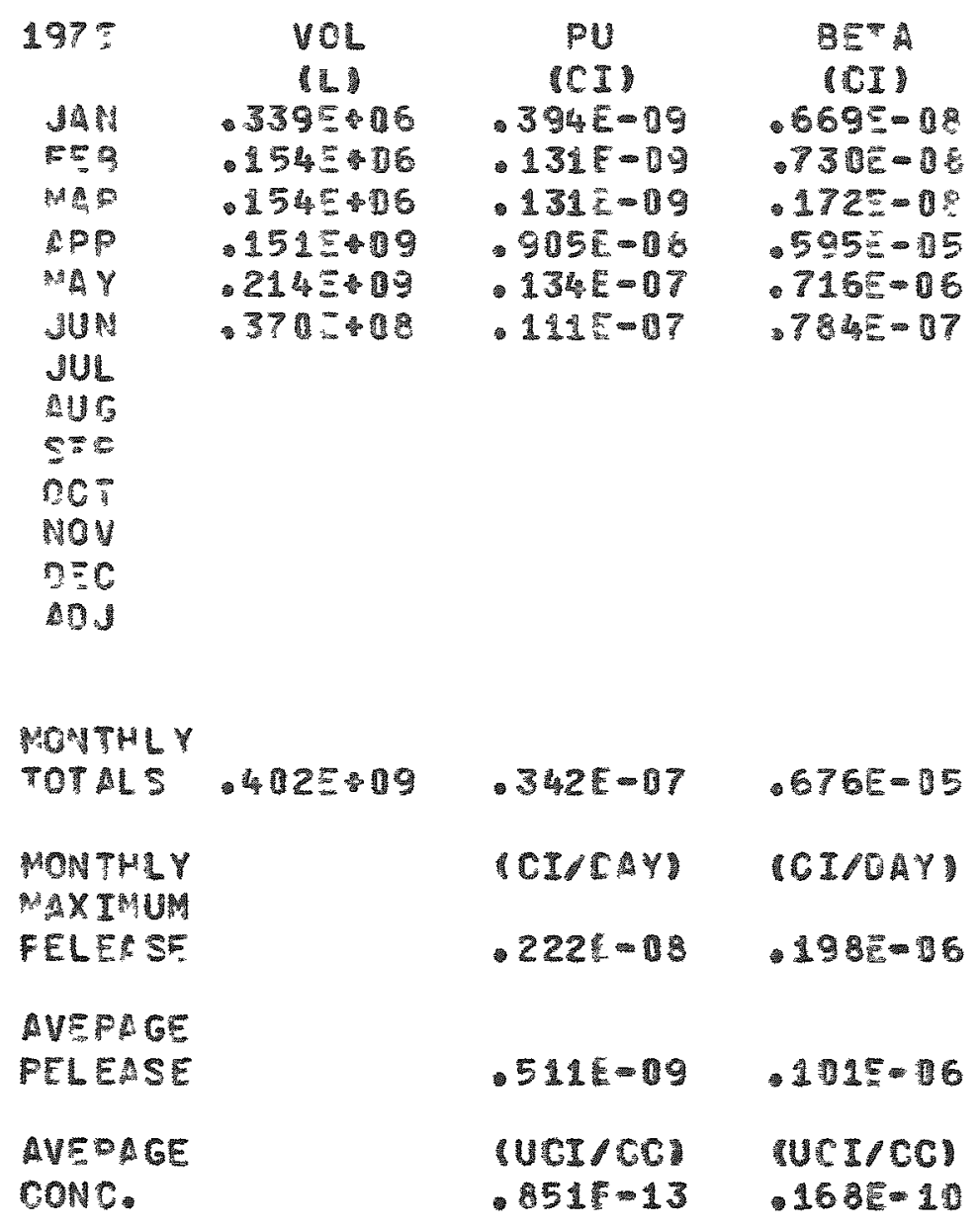

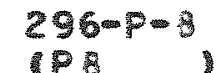

\begin{tabular}{|c|c|c|}
\hline $\begin{array}{l}\text { YOL } \\
\text { IL }\end{array}$ & $\begin{array}{c}\text { FU } \\
\text { IGI }\end{array}$ & $\begin{array}{l}3.74 \\
001\end{array}$ \\
\hline 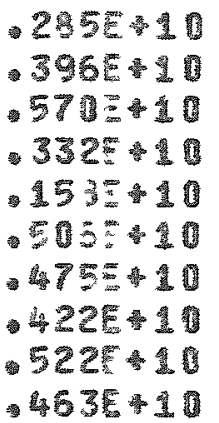 & 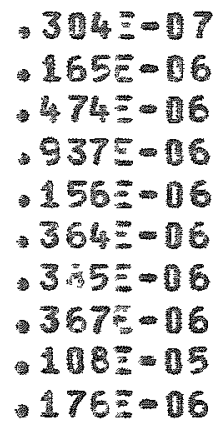 & 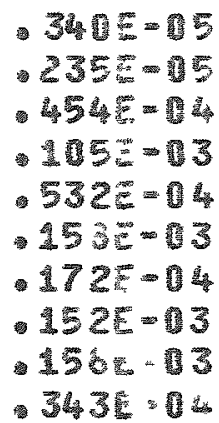 \\
\hline
\end{tabular}

\begin{tabular}{|c|c|c|}
\hline \multirow[t]{5}{*}{ 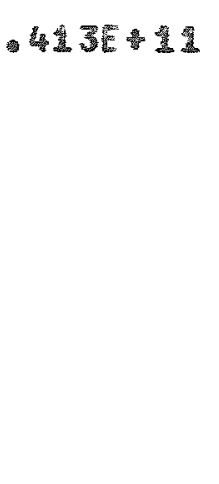 } & - $435=05$ & $.729 E-13$ \\
\hline & SIIDAY & (OT/OAY) \\
\hline & $-465=07$ & 59205 \\
\hline & $-157 \pm=07$ & $.276=05$ \\
\hline & $\begin{array}{l}\text { UCI/CS } \\
-100=12\end{array}$ & $\begin{array}{l}1001 / 00 \\
177 E .10\end{array}$ \\
\hline
\end{tabular}




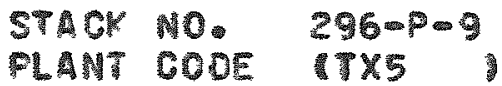

FACILITY 2H2-TX WASTE EVAP.

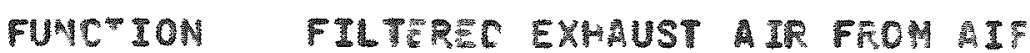
COOLING OF CONCENTPATE RECEIUING TANKS IN TX FARM 113,114, ANO $115-7 X$.

\begin{tabular}{|c|c|c|c|}
\hline 1975 & $\begin{array}{l}\text { VOL } \\
\text { ML }\end{array}$ & $\begin{array}{l}\text { PU } \\
\text { COSI }\end{array}$ & $\begin{array}{l}\text { 9ETA } \\
\text { ICI }\end{array}$ \\
\hline 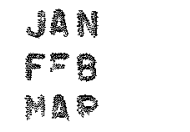 & 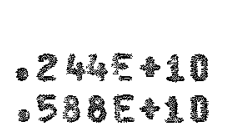 & $\begin{array}{l}174 E-17 \\
.693 E-16\end{array}$ & $\begin{array}{r}2115-15 \\
-1375=04\end{array}$ \\
\hline $\begin{array}{l}\text { APR } \\
\text { MPY }\end{array}$ & & & \\
\hline $\begin{array}{l}\text { IUN } \\
\text { MUL } \\
406\end{array}$ & 2:5s & $-435 F=0$ & $-494=06$ \\
\hline 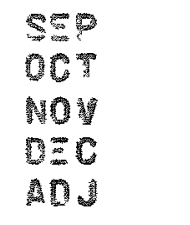 & $-732-109$ & $.793-16$ & $-699=16$ \\
\hline MONTHEY & & & \\
\hline TOTHS & $-927 \leq 10$ & $-157 E=15$ & $-270 E=0 \%$ \\
\hline $\begin{array}{l}\text { MONTHLY } \\
\text { MXIVUM }\end{array}$ & & SCIACYY & (CINOAY \\
\hline FELESSF & & $.261 E-16$ & $.733=6$ \\
\hline $\begin{array}{l}\text { AVERGE } \\
\text { RELESSE }\end{array}$ & & $-43-67$ & $-546-6$ \\
\hline $\begin{array}{l}\text { AVERGE } \\
\text { CONC. }\end{array}$ & & $\begin{array}{l}\text { UCI/CC } \\
-162 E=12\end{array}$ & $\begin{array}{l}\text { UCF/CC } \\
-183-11\end{array}$ \\
\hline
\end{tabular}

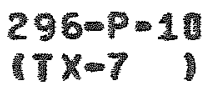

24 2-TX HASTE EVAP.

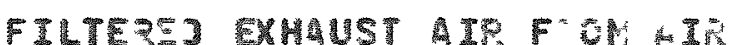

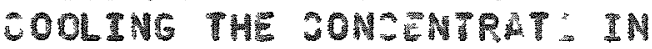
TX FARH BOTTMS TANKS

(110, 11. ANO 112-TX).

\begin{tabular}{|c|c|c|}
\hline 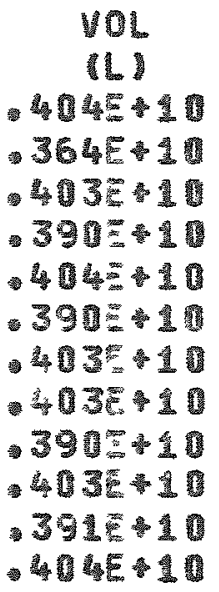 & 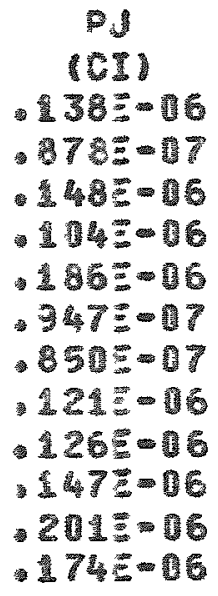 & 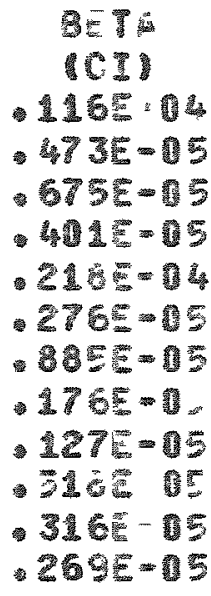 \\
\hline
\end{tabular}

\begin{tabular}{|c|c|c|}
\hline \multirow[t]{5}{*}{$475=11$} & $.162=-15$ & $.7465-04$ \\
\hline & SEIOAY & ICI/OAY \\
\hline & $.5705=00$ & $.703 \bar{E}-06$ \\
\hline & $-42=-08$ & $-204 E-06$ \\
\hline & $\begin{array}{l}\text { UCE/CO } \\
340=-13\end{array}$ & $\begin{array}{l}\text { UCI/CG } \\
.157 E-11\end{array}$ \\
\hline
\end{tabular}


STACK HO. 296-P-11

PLANT COOF

FACILITY 242-TX WASTE EVAD.

FUNCTION FILTEREO EXHAUST AIR FOOM COOLING THE CONC-NTRATE IN TX FAR EOT TOHS TANK $113-$ T

\begin{tabular}{|c|c|c|c|}
\hline 1975 & $\begin{array}{l}\text { VOL } \\
\text { IL }\end{array}$ & $\begin{array}{c}\text { PU } \\
\text { (SII }\end{array}$ & $\begin{array}{l}\text { BET } \\
\text { BII }\end{array}$ \\
\hline $34 N$ & $.192{ }^{2}+199$ & $-181 E-18$ & $-620-17$ \\
\hline & $.173=09$ & $-178 E-08$ & $-129 E-16$ \\
\hline$M R$ & $.1925+19$ & $-391 E-18$ & $.184 E=06$ \\
\hline AP & 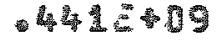 & $.753=-103$ & $-2275-06$ \\
\hline Hat & -442509 & $.728 E-06$ & $-209 E-16$ \\
\hline JUN & 040109 & - 876E-0 & $\begin{array}{l}360=-1 \\
25\end{array}$ \\
\hline ive & $456 E+09$ & 10 & \\
\hline AU & $-405=+09$ & $-100 E-07$ & $155 E-06$ \\
\hline$S=P$ & $-235=109$ & $.738 E-08$ & $.7555-10$ \\
\hline DC & $189^{2}+199$ & $-576=0$ & $-2712-16$ \\
\hline Nov & $-128=09$ & $-6726=0$ & $.6925=7$ \\
\hline 250 & $1045+09$ & $.369 \mathrm{~g}=0$ & $.683=7$ \\
\hline
\end{tabular}

MONTHLY

\begin{tabular}{|c|c|c|c|}
\hline TOTALS & 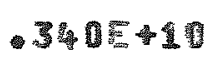 & $-8114-97$ & $.533 E=0$ \\
\hline MCNTHLY & & TCI/DAV & ICIDOAY \\
\hline $\begin{array}{l}\text { MXIVUM } \\
\text { HLEASE }\end{array}$ & & - 348E-D9 & $-127 E-06$ \\
\hline $\begin{array}{l}\text { AVERLGE } \\
\text { PFL CASE }\end{array}$ & & $-222 E-19$ & $1465-17$ \\
\hline $\begin{array}{l}\text { AEOAGE } \\
\text { CONC. }\end{array}$ & & $\begin{array}{l}\text { UCI/CCI } \\
-2392-13\end{array}$ & $\begin{array}{l}\text { UCI/CC } \\
.157-11\end{array}$ \\
\hline
\end{tabular}

$296-P-12$
$7 x=9$

242-TX WASTE EVAP.

FILTERED EXHLUST AIR FHOM COOLING THE SONCENTRAT: IN TX FAPRS SOTTOMS THNS 1102, 105. AN

\begin{tabular}{|c|c|c|}
\hline 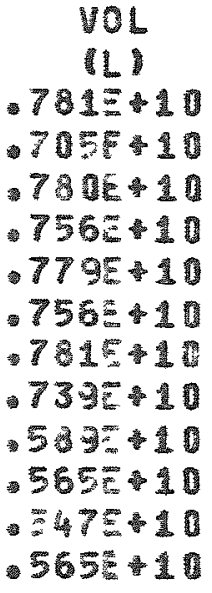 & 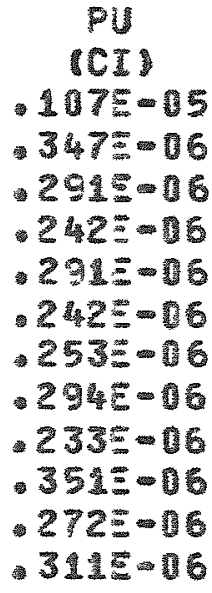 & 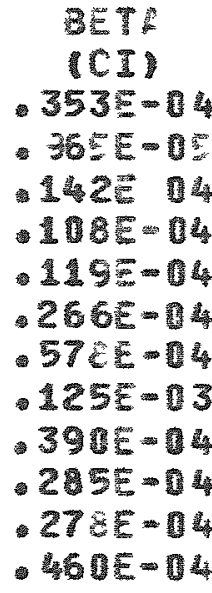 \\
\hline
\end{tabular}

\begin{tabular}{|c|c|c|}
\hline \multirow{5}{*}{634581} & $-42 n=-05$ & $.4325=03$ \\
\hline & GIJDAY & COIDAY \\
\hline & $-345=-67$ & $-403 E-05$ \\
\hline & $-115=07$ & $-118 F-05$ \\
\hline & $\begin{array}{l}\text { UCI CCI } \\
.503 E-13\end{array}$ & $\begin{array}{l}\text { UCI/COI } \\
518 E-11\end{array}$ \\
\hline
\end{tabular}


STACK NO. $296-8-14$

PLANT COOF ODETK15)

FACILITY 241-S TANK FAPH

FUNCTION FILTERED EXLUST ATR FROM TH 量1. 12.103,10\%.105 ANO 106-S TANKS.

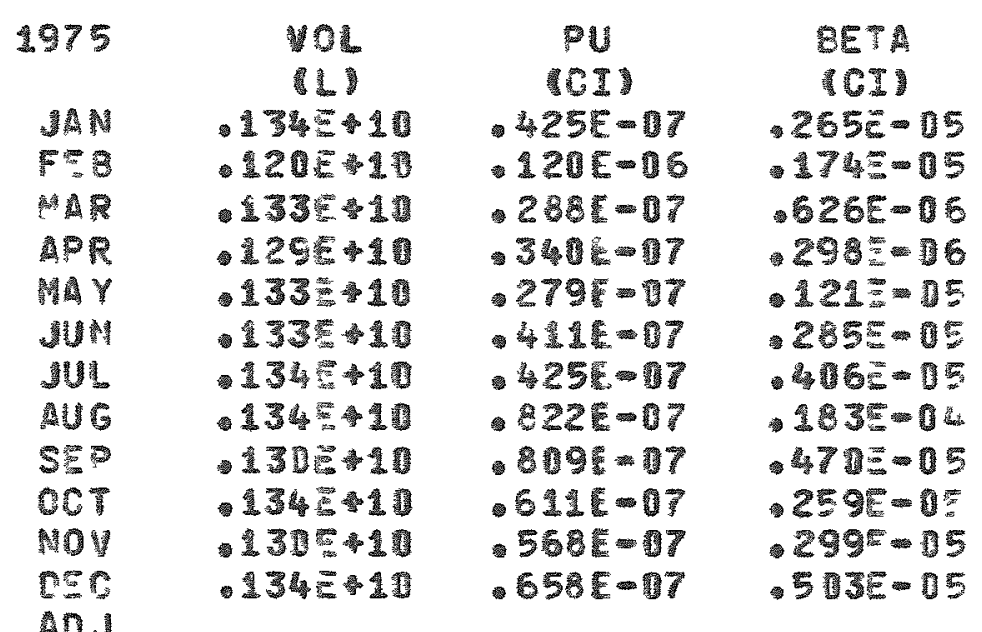

MONUTHLY

\begin{tabular}{|c|c|c|c|}
\hline ToTh & $158=11$ & $.6845=06$ & 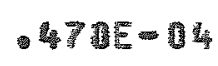 \\
\hline 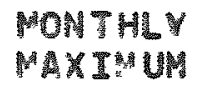 & & (CI/CAVI) & TCIOOAY \\
\hline FELEASE & & $-429 E-08$ & .610505 \\
\hline 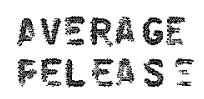 & & $.188=08$ & $-135=-16$ \\
\hline $\begin{array}{l}\text { AVEFG GE } \\
\text { CONC. }\end{array}$ & & $\begin{array}{l}\text { UCI/CCI } \\
-433 E-13\end{array}$ & $\begin{array}{l}\text { UCI/CC } \\
298 E-11\end{array}$ \\
\hline
\end{tabular}

\section{$296-p=15$ \\ DETK25}

241-S A A FAR

FILTEES EXAUST AR FOON THE 107.103.109.10.11 GMC 112-3

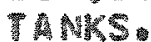

\begin{tabular}{|c|c|c|}
\hline 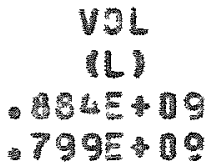 & 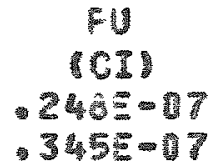 & $\begin{array}{c}\text { 8.T } \\
101 \\
-299=04 \\
-45=05\end{array}$ \\
\hline 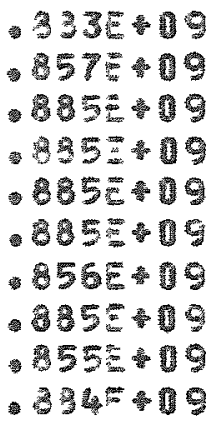 & 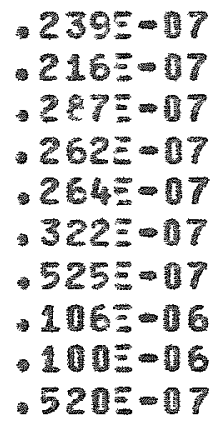 & 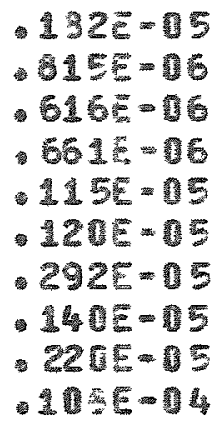 \\
\hline
\end{tabular}

\begin{tabular}{|c|c|c|}
\hline \multirow[t]{5}{*}{104551} & $.529=-06$ & - $582 E=04$ \\
\hline & 13IOAAY & OCL $/ 0 \mathrm{MY}$ \\
\hline & $343=-18$ & $.965 E=06$ \\
\hline & $145=-06$ & $-1595=06$ \\
\hline & $\begin{array}{l}1002 / 031 \\
.507=-13\end{array}$ & $\begin{array}{l}\text { UCE/CO } \\
-557 E=11\end{array}$ \\
\hline
\end{tabular}


STACK NO. $\quad 296-P=16$

PLANT CODE 1030 ।

FACILITI $B$ TANK FAFM

FUNCTION FILTERPD EXHAUST AIR FROM THE 101,103, ANC 106-B TANKS

\begin{tabular}{|c|c|c|c|}
\hline 1975 & $\begin{array}{c}\text { VDL } \\
7 L 1 \\
.594 .10\end{array}$ & $\begin{array}{c}\text { PU } \\
\text { CCI } \\
548=16\end{array}$ & $\begin{array}{c}\text { BET } \\
\text { CTI } \\
546 E-15\end{array}$ \\
\hline $\begin{array}{l}F=B \\
M A R\end{array}$ & $\begin{array}{l}5375.12 \\
.5955 .11\end{array}$ & $\begin{array}{l}.310=06 \\
.613=06\end{array}$ & $\begin{array}{l}3995-05 \\
.122 z-04\end{array}$ \\
\hline ADs & $.574=0$ & $.580 \varepsilon=76$ & $.653=-05$ \\
\hline 政 & $.387+109$ & $.975 E=07$ & $.759 E=06$ \\
\hline $\begin{array}{l}\text { JUA } \\
\text { JUL } \\
\text { MUG } \\
\text { SED }\end{array}$ & & & \\
\hline OCT & $.402 E+10$ & $.1992=06$ & $-104 E-15$ \\
\hline $\begin{array}{l}\text { MV } \\
\text { DEC }\end{array}$ & $\begin{array}{l}2595.10 \\
.1185 .10\end{array}$ & 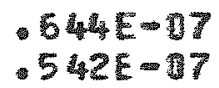 & $\begin{array}{l}591 E-16 \\
302 E-16\end{array}$ \\
\hline
\end{tabular}

MONTHLY

\begin{tabular}{|c|c|c|c|}
\hline TOTh & 312511 & $.247 c-05$ & -395-14 \\
\hline NONTHLY & & ICI/CAY & (CI/OAY) \\
\hline MXTMUN & & & \\
\hline FELEASE & & - $488 E-17$ & $394 E-06$ \\
\hline $\begin{array}{l}\text { AVSRAB } \\
\text { FELEASE }\end{array}$ & & $-1215=77$ & $-152 \equiv=06$ \\
\hline AVEPGE & & UCIACG & TUCT/CC \\
\hline CONC. & & $-7915-13$ & $.991 E-12$ \\
\hline
\end{tabular}

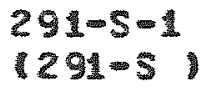

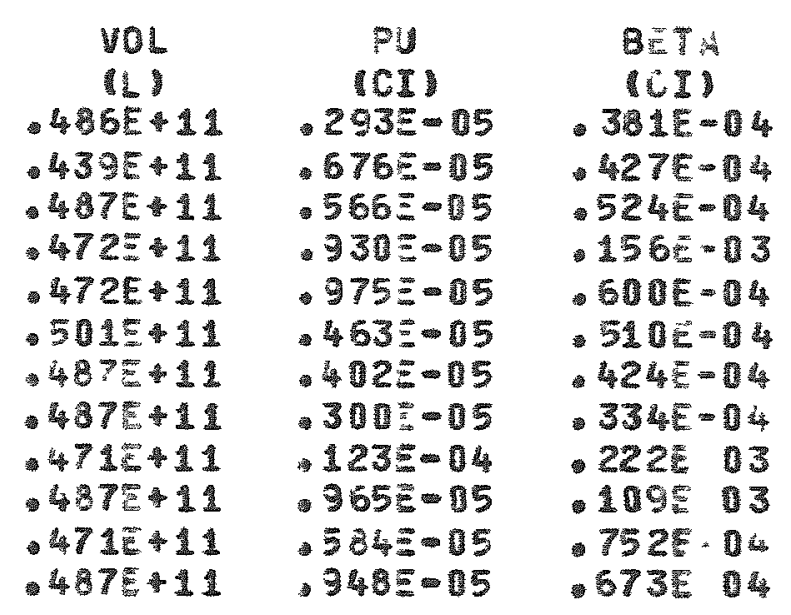

\begin{tabular}{|c|c|c|}
\hline \multirow[t]{5}{*}{$=737+12$} & $834 E-04$ & $.9505 \cdot 03$ \\
\hline & (CI/OAY) & (CI/DAY \\
\hline & $-4=-06$ & $\cdot 741 E-05$ \\
\hline & $-228=06$ & $.2605-05$ \\
\hline & $\begin{array}{l}(J C I / C 3) \\
-145 \equiv-12\end{array}$ & $\begin{array}{l}\text { UCI/CCI } \\
.165 \mathrm{E}=11\end{array}$ \\
\hline
\end{tabular}




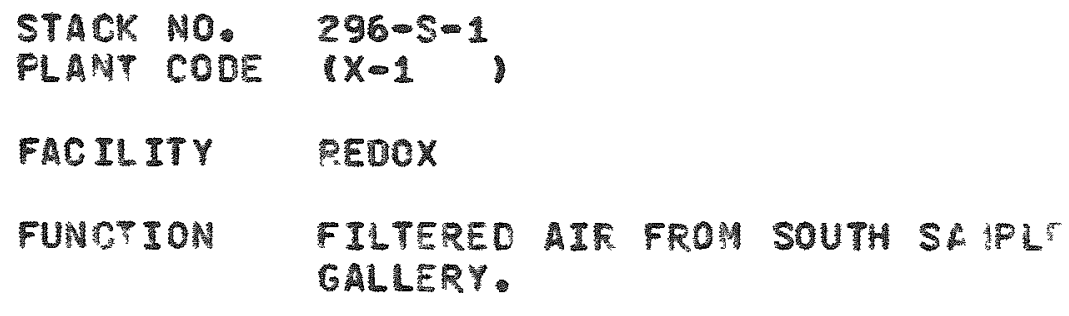

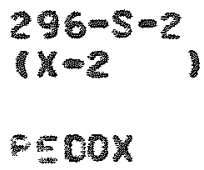

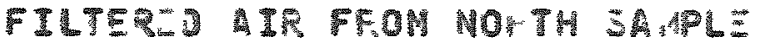
GALLEY AMD SAMPLSR HOODS.

\begin{tabular}{|c|c|c|c|}
\hline 1975 & $\begin{array}{l}\text { VOL } \\
\text { (1) }\end{array}$ & $\begin{array}{c}\text { PU } \\
\text { CUI }\end{array}$ & $\begin{array}{l}\text { BETA } \\
\text { MCII }\end{array}$ \\
\hline ARN & $.293=10$ & - $139 E-06$ & $.1615-05$ \\
\hline $\mathrm{FE}$ & 264510 & $.277 E-06$ & $2005-05$ \\
\hline MA & $.293 \div-110$ & $.9875-17$ & $149 E-1$ \\
\hline$A P P$ & $29=10$ & $.295 E=06$ & $6305-0$ \\
\hline HY & $.298=10$ & $143 E=06$ & $-114 E-0$ \\
\hline JUN & $294=10$ & $.395-05$ & $262^{-1}-1$ \\
\hline SUL & 2935 & $.548 c-66$ & $34=0$ \\
\hline AUG & -293E.10 & $15-16$ & \\
\hline$S^{* p}$ & 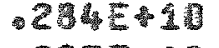 & $.730 E-66$ & $5=-0$ \\
\hline ost & 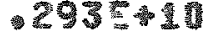 & - $942=05$ & $.1045=0$ \\
\hline Nov & $.2844^{6} \cdot 10$ & $-402-06$ & $.992 \equiv=0$ \\
\hline$n=0$ & 2935 & $.520 E-06$ & 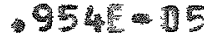 \\
\hline 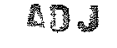 & & & \\
\hline
\end{tabular}

\begin{tabular}{|c|c|c|}
\hline $\begin{array}{l}\text { VOL } \\
\text { (L) }\end{array}$ & $\begin{array}{l}\text { PU } \\
\text { 10I }\end{array}$ & $\begin{array}{l}\theta_{-}=4 \\
\text { (c) }\end{array}$ \\
\hline $2192=10$ & $993 z-07$ & $.228: 06$ \\
\hline 195 & $-116=-06$ & .645 .15 \\
\hline 2135 & $132=016$ & .2555 \\
\hline -21皇: & $-127=75$ & -156 - 0 \\
\hline $219=10$ & $137=-06$ & 13385 \\
\hline $2195+10$ & $-195=76$ & $.926 E=08$ \\
\hline 210510 & $145=06$ & 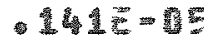 \\
\hline $213=10$ & $1125=6$ & $-557=96$ \\
\hline $212 k+10$ & - $139=06$ & 31 sE $=05$ \\
\hline 215 & $147=-16$ & $1785=05$ \\
\hline & - $38=-17$ & \\
\hline & & \\
\hline
\end{tabular}

MONTHLY

\begin{tabular}{|c|c|c|c|}
\hline Totals & -346811 & $-485=85$ & $.68 D E-74$ \\
\hline 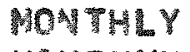 & & DOITAYI & CDIOAY \\
\hline 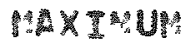 & & & \\
\hline FELSLS & & $.304 E=77$ & $-3525=05$ \\
\hline $\begin{array}{l}\text { AVE SGE } \\
\text { OFLESSE }\end{array}$ & & $134-07$ & $.186=06$ \\
\hline $\begin{array}{l}\text { MVER:GE } \\
\text { CoNe. }\end{array}$ & & $\begin{array}{l}\text { UST/COL } \\
141-12\end{array}$ & $\begin{array}{r}\text { UCI/CC } \\
-196 \mathrm{E}-11\end{array}$ \\
\hline
\end{tabular}

\begin{tabular}{|c|c|c|}
\hline \multirow[t]{5}{*}{$2538+1$} & $149 \equiv=15$ & $156=04$ \\
\hline & TEI/DAY & TCS/DHV \\
\hline & $497=08$ & 108506 \\
\hline & - 400 & $4285-07$ \\
\hline & $\begin{array}{l}\text { UCI } \\
.573=13\end{array}$ & $\begin{array}{l}\text { UCE/ACI } \\
.605=-12\end{array}$ \\
\hline
\end{tabular}




\begin{tabular}{|c|c|}
\hline $\begin{array}{l}\text { STACK NO. } \\
\text { PLAN CODE }\end{array}$ & $\begin{array}{l}296-5-4 \\
e F-3\end{array}$ \\
\hline FACILITY & EDOX \\
\hline FUNC ION & 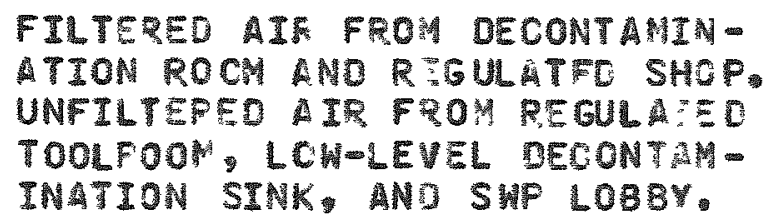 \\
\hline
\end{tabular}

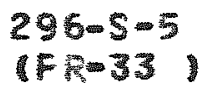

\begin{tabular}{|c|c|c|c|}
\hline 1975 & $\begin{array}{l}\text { YOL } \\
1183\end{array}$ & $\begin{array}{c}\text { PU } \\
\text { COII }\end{array}$ & $\begin{array}{l}\text { BE } \\
\text { 1CII }\end{array}$ \\
\hline SN & $.616 E \div 10$ & $-124 E-15$ & $1195-14$ \\
\hline$F E$ & - 556? 10 & $.996-06$ & $-124 E-14$ \\
\hline$M A R$ & 514510 & $.7475-06$ & $.9905-07$ \\
\hline$A D R_{0}$ & $.596-10$ & $-1345-05$ & 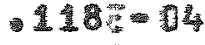 \\
\hline My & $.615=10$ & $.125 E-05$ & $149 \varepsilon=04$ \\
\hline 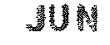 & .614517 & $\cdot 836-06$ & $132=04$ \\
\hline 3UL & 614510 & $-161 E-05$ & $-2085=04$ \\
\hline A116 & 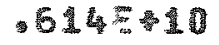 & $.132 x-5$ & $.1905=14$ \\
\hline$S E P$ & $595=10$ & $.131 E=05$ & $.279 E-D 4$ \\
\hline 09 & 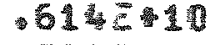 & $-143 E-15$ & $.2315-04$ \\
\hline NOV & 594510 & $.720 E-06$ & $154=-14=$ \\
\hline tec & $61.62=10$ & $.735 E=16$ & $151=-104$ \\
\hline 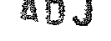 & & & \\
\hline
\end{tabular}

MONTHLY

\begin{tabular}{|c|c|c|c|}
\hline TOTALS & - $7266^{2}$ & $-133 E-04$ & $.195=03$ \\
\hline MONTHLV & & 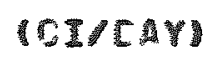 & CEADAY \\
\hline MAXIMUN & & & \\
\hline$F=L S E$ & & $-519 E=07$ & $.93 B E-76$ \\
\hline $\begin{array}{l}\text { AVEPGE } \\
\text { TLEDSE }\end{array}$ & & $-365 c-07$ & $-535 E-76$ \\
\hline $\begin{array}{l}\text { AVEPAGE } \\
\text { CONC. }\end{array}$ & & $\begin{array}{l}\text { UCI/COL } \\
184-12\end{array}$ & $\begin{array}{l}\text { UCI/CC } \\
269 E-11\end{array}$ \\
\hline
\end{tabular}

\begin{tabular}{|c|c|c|}
\hline 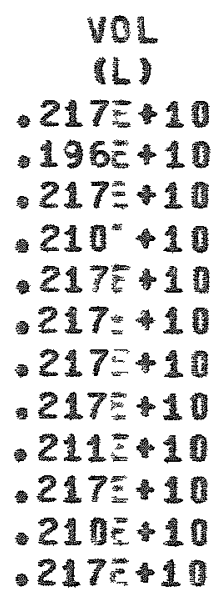 & 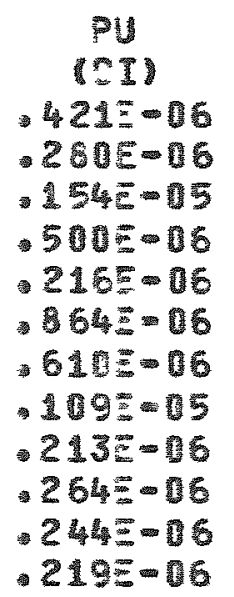 & 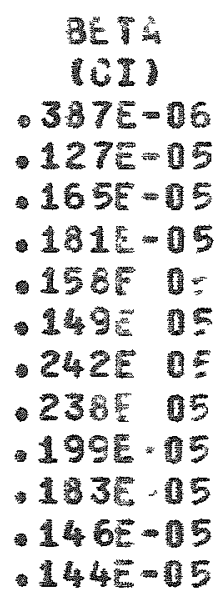 \\
\hline
\end{tabular}

\begin{tabular}{|c|c|c|}
\hline \multirow[t]{5}{*}{$257=11$} & $.646 z=05$ & 197E- \\
\hline & ISIOAY & (CD/OAY) \\
\hline & $-497=-107$ & -782E-07 \\
\hline & $-177=07$ & - 540 \\
\hline & $\begin{array}{l}\text { UUCI/CE } \\
252=12\end{array}$ & $\begin{array}{l}\text { UUCI/CC } \\
-768 E 12\end{array}$ \\
\hline
\end{tabular}




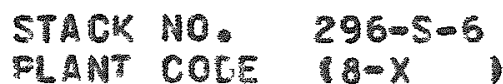

FACILITY PEOX

FUMOTIOH UNFILTESSO AIZ FROM THE STLO GALLETY, OQGANIC FEED TANK, AND SEMPLE CLEUATOR.

\begin{tabular}{|c|c|c|c|}
\hline 1975 & 100 & $\begin{array}{c}P U \\
\text { CII }\end{array}$ & 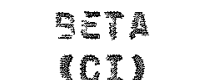 \\
\hline J率算 & $-116^{2}+11$ & $-234-85$ & $280=0 L$ \\
\hline 55 & $.1055^{2} \cdot 11$ & $180-75$ & . $2868=04$ \\
\hline A & $-115=412$ & $-180 \varepsilon-15$ & $2945=14$ \\
\hline 283 & 1125 & $-336 c-25$ & $.573 E-03$ \\
\hline$\gamma$ & 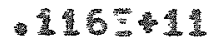 & $-168 E=75$ & $2785=04$ \\
\hline UU篗 & $119=11$ & $.160=-15$ & $2475=04$ \\
\hline IIL & 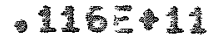 & $-257 E-15$ & .3215 \\
\hline 806 & $-116=11$ & $172 E=05$ & $9 E=04$ \\
\hline 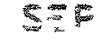 & $112=11$ & $-314+4=05$ & $-451 E=0$ \\
\hline cos & 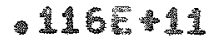 & $-170 E-0 z$ & $.3605=04$ \\
\hline AOV & 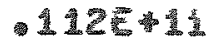 & $-1856=05$ & $2513-1.4$ \\
\hline$p=c$ & 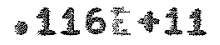 & $-127=05$ & $2565=04$ \\
\hline & & & \\
\hline
\end{tabular}

\section{FONTHLY}

\begin{tabular}{|c|c|c|c|}
\hline TOTALs & 1375 준 12 & $.2495-14$ & $.907=03$ \\
\hline 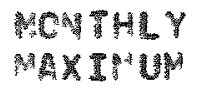 & & ICI/CIMI & COLOHY \\
\hline PFLASE & & $-112 E-16$ & $191 E-04$ \\
\hline $\begin{array}{l}\text { AVERAGE } \\
\text { RELEISE }\end{array}$ & & $.683 E=07$ & $-2495-05$ \\
\hline $\begin{array}{l}A V=\text { GE } \\
\text { Coñ. }\end{array}$ & & $\begin{array}{l}\text { (UCI/CC } \\
182 E-12\end{array}$ & $\begin{array}{l}\text { VOCI/CG } \\
.662 E-11\end{array}$ \\
\hline
\end{tabular}

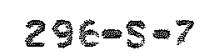

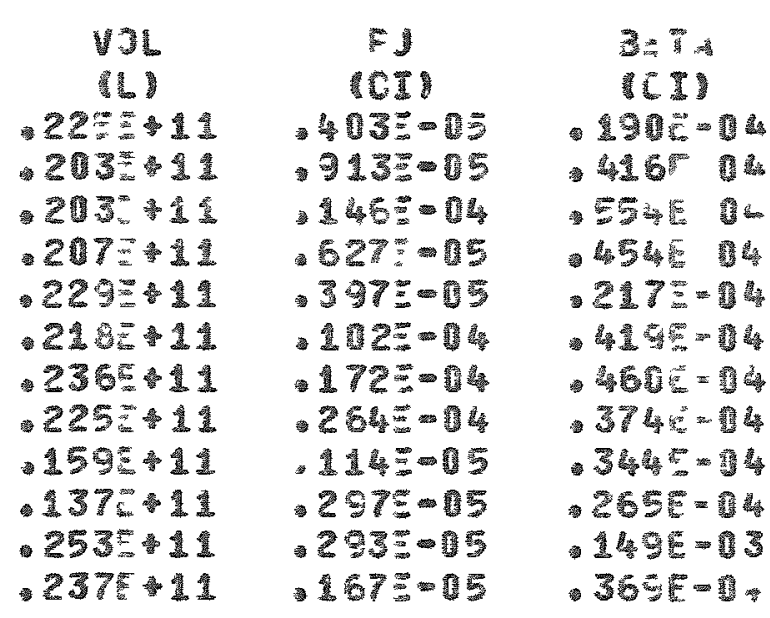

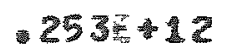


STACV NO. $296-5-13$

FACILITY 222-5 L COPATORY

FUNGTION FILTERE AIF FROM HOOOS GLVE BOXES, HOT CELLS, ANC VENTILAT ION.

\begin{tabular}{|c|c|c|c|}
\hline 1975 & $\begin{array}{l}\text { 10L } \\
\text { (1) }\end{array}$ & $\begin{array}{l}\mathrm{PU} \\
\mathrm{CI}\end{array}$ & $\begin{array}{l}\text { Befa } \\
\text { ICI }\end{array}$ \\
\hline$M A N$ & $-931=-11$ & $510=-15$ & $-269-3$ \\
\hline$F=8$ & - $833 E+11$ & $-298=05$ & -451E-03 \\
\hline $\begin{array}{l}y^{2} \\
420\end{array}$ & $\begin{array}{r}9785-11 \\
948\end{array}$ & $\begin{array}{r}345 E-05 \\
331 E=15\end{array}$ & $\begin{array}{l}159=-13 \\
201=-13\end{array}$ \\
\hline MY & $.978 E+1$ & $.291 E-05$ & $-2435=03$ \\
\hline JUN & .978511 & $.305 z-05$ & $614=0$ \\
\hline Sub & $.978 \leq+11$ & $.2478-155$ & 168 \\
\hline 046 & $978 E+11$ & $.235=-15$ & 1 \\
\hline Sep & $9475+11$ & - $242 E=05$ & $-347=-0$ \\
\hline Oct & $.978=11$ & $-275-05$ & $.4315-0$ \\
\hline Mov & $947 E-11$ & $-264 E=05$ & $.874=-0$ \\
\hline $\mathrm{CFC}$ & $.994 z^{2} 1$ & $-329-05$ & 200 \\
\hline
\end{tabular}

MONTHLY

\begin{tabular}{|c|c|c|c|}
\hline TOTALS & $116 E^{13}$ & $-367 E-14$ & $.360 E=12$ \\
\hline $\begin{array}{l}\text { MONTHLY } \\
\text { M X IMUM }\end{array}$ & & |CI/DAY & CI/CAY \\
\hline FCLESE & & $.165 E=00$ & $-291=-14$ \\
\hline \multicolumn{4}{|l|}{ AVSOG } \\
\hline PELEASE & & $-1011-06$ & $.986 z-05$ \\
\hline MVPSES & & UCI/CO & IUCI/CC \\
\hline CONC. & & $-318 z-13$ & $312 E-11$ \\
\hline
\end{tabular}

$296-5-14$

$(s-14)$

$241-5 \times$ T THK FAPH

FIITERED NONSONOENSIBL YAPONS FPOH WAST" STORAGE TANS IM 24.1-SX TANK FARH

\begin{tabular}{|c|c|c|}
\hline 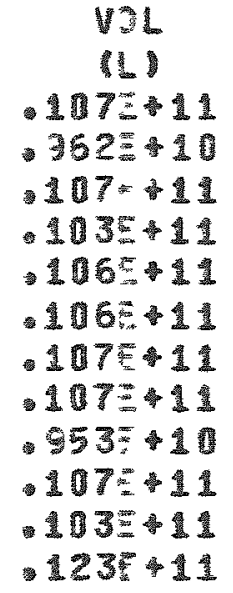 & 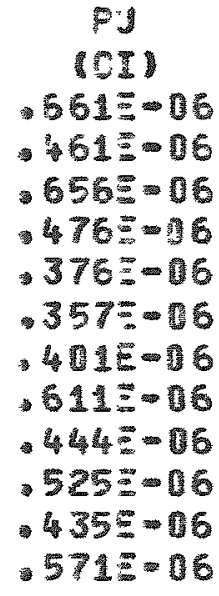 & 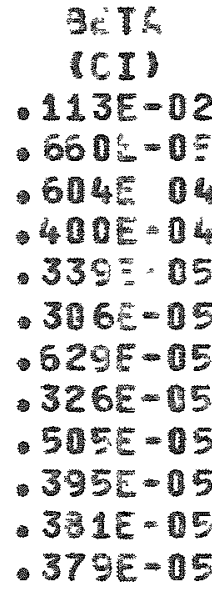 \\
\hline
\end{tabular}

\begin{tabular}{|c|c|c|}
\hline \multirow{5}{*}{$127=12$} & $.3975-05$ & $1275 \cdot 02$ \\
\hline & ISIODAY & (CI/DAY) \\
\hline & $213=-07$ & $-365=04$ \\
\hline & $1645-07$ & $.3435=05$ \\
\hline & $\begin{array}{l}\text { UCI/CE } \\
.42=-13\end{array}$ & $\begin{array}{l}\text { (UCD/CC) } \\
-100=10\end{array}$ \\
\hline
\end{tabular}




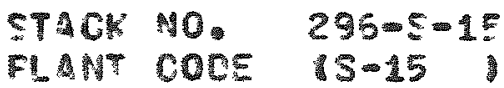

FACILITY 241-5X TANK FAPM

FUNCION FILTESE EXHAUST ATE FPOH COOLING OF CRIDC SLUDGE IH:X FARM THNKS 107, 108,109 . ANC $112-5 \times$.

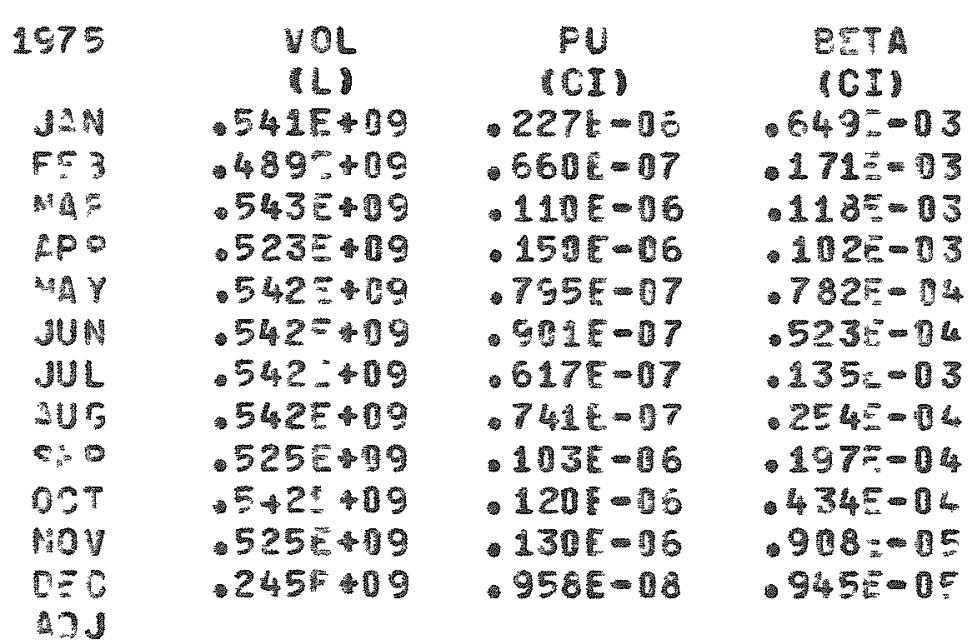

Manthelly

TOTALS $610 \div 10$

\begin{tabular}{|c|c|c|c|}
\hline Totals & 6110 & $122=5$ & - $141^{2}=2$ \\
\hline MOVTHEY & & TrIRAAY & (CIDJAY \\
\hline : & & & \\
\hline F6L & & $.732 E-08$ & $.2095-04$ \\
\hline 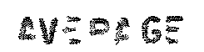 & & & \\
\hline$F=L S$ & & $351 E-78$ & - $405-05$ \\
\hline $\begin{array}{l}\text { WE-GE } \\
\text { COUC. }\end{array}$ & & UOA/CC & UUCI/CO \\
\hline Conc. & & $.200 E-12$ & $.231=-69$ \\
\hline
\end{tabular}

$296-S-16$ $(219-5)$

\section{2-S LAE ST3GG:}

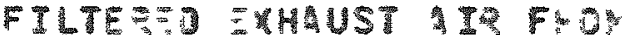

291-5 VAULT 品NC WAST MAKS.

\begin{tabular}{|c|c|c|}
\hline 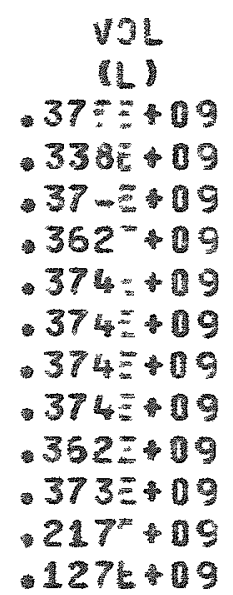 & 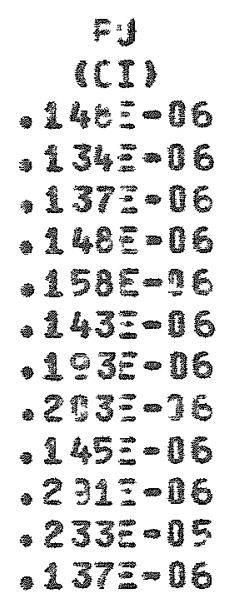 & 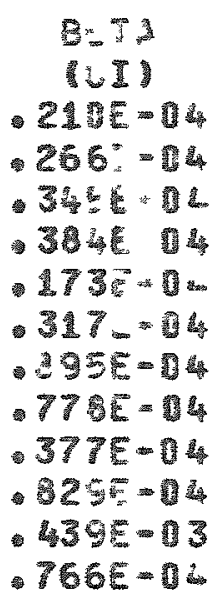 \\
\hline
\end{tabular}

\begin{tabular}{|c|c|c|}
\hline \multirow[t]{5}{*}{$-403 \bar{E}-10$} & $-47 \pm 0.5$ & $.973:-03$ \\
\hline & SEIOAYI & (CI/D. \\
\hline & $.77=-67$ & $.146 E-04$ \\
\hline & $-114=-07$ & $267 E-15$ \\
\hline & $\begin{array}{l}\text { UCI/CO) } \\
103=-11\end{array}$ & $\begin{array}{l}1 \text { UCI }=01 \\
2425=09\end{array}$ \\
\hline
\end{tabular}


$\begin{array}{ll}\text { STACK NOE } & 296-5-18 \\ \text { PUAN CODE VOTKR }\end{array}$

FAOILITY 242-5 WASE SVAP.

FUNC ION FILT:RED ATR FRO THE 242-3

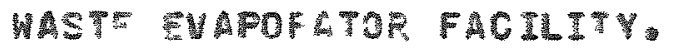

\begin{tabular}{|c|c|c|c|}
\hline 1975 & 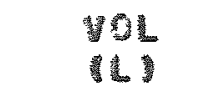 & $\begin{array}{c}\text { PU } \\
\text { COII }\end{array}$ & $\begin{array}{l}35 \mathrm{~A} \\
1010\end{array}$ \\
\hline 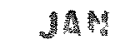 & 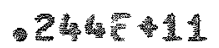 & $.569=06$ & $-279=02$ \\
\hline$F=3$ & $221 \mathrm{E}$ & $-4374=06$ & $932=15$ \\
\hline$\Rightarrow$ & $2^{2} 44=11$ & $-113 E=05$ & $-609-05$ \\
\hline$A=$ & -236511 & $118 E=05$ & $.629=05$ \\
\hline 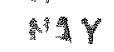 & 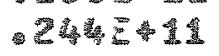 & $-134 c=5$ & $100=-14$ \\
\hline 3U & - $2445-14$ & - $7496-6$ & $-753^{3}=25$ \\
\hline UUL & 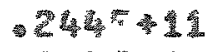 & $.992 x-06$ & $189=05$ \\
\hline AUG & 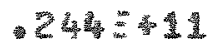 & $-1+12=05$ & $-251=-15$ \\
\hline $5: p$ & $.236=11$ & - $130 c-15$ & $-929 \%-05$ \\
\hline ort & 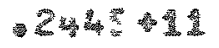 & $-171+06$ & - $100=-14$ \\
\hline ing & $-236 .+11$ & - $133-135$ & $-832=-75$ \\
\hline & 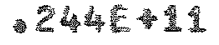 & $-1111-05$ & $=05$ \\
\hline
\end{tabular}

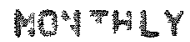

TOTALS

MXThum

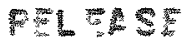

WE

PELESE

AV

conc.

\begin{tabular}{|c|c|c|}
\hline \multirow[t]{5}{*}{.2885 .12} & $-123=4$ & $-122=3$ \\
\hline & (CI/CAYI & ICIDAM \\
\hline & $.553 E-97$ & $-333 E-06$ \\
\hline & - 371 $2-07$ & $.367=-06$ \\
\hline & $\begin{array}{l}\text { UGI/CCI } \\
-429 E-13\end{array}$ & $\begin{array}{l}\text { UCI/CC } \\
-25=-12\end{array}$ \\
\hline
\end{tabular}

\section{$296-5-19$ \\ IPESS}

242-S WASTE EUAP.

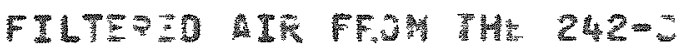
CELL AOH VESSEL VENV

\begin{tabular}{|c|c|c|}
\hline 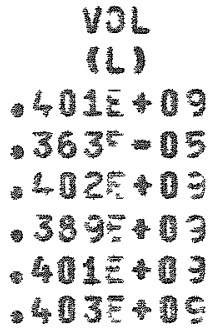 & 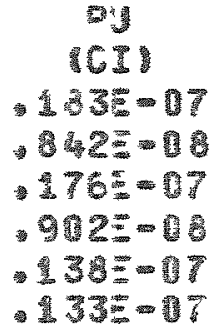 & 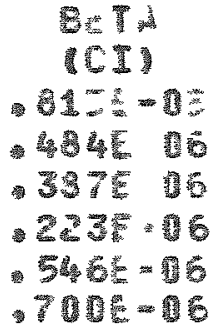 \\
\hline 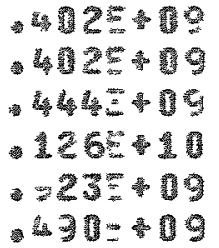 & 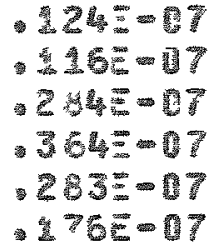 & 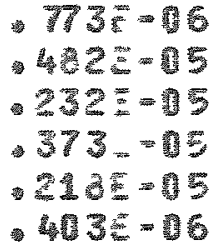 \\
\hline
\end{tabular}

\begin{tabular}{|c|c|c|}
\hline \multirow[t]{5}{*}{$532=10$} & $-215=06$ & 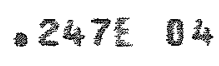 \\
\hline & 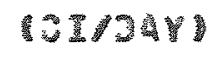 & TCD $10 \times$ \\
\hline & $.1175-68$ & $-2635-16$ \\
\hline & $.593=19$ & $.6018-17$ \\
\hline & $\begin{array}{l}\text { UCI/COI } \\
363 \pm-13\end{array}$ & $\begin{array}{l}\text { UCI/DG) } \\
-415=11\end{array}$ \\
\hline
\end{tabular}




\begin{tabular}{|c|c|}
\hline $\begin{array}{l}\text { STACY NO } \\
\text { PLANT CODE }\end{array}$ & $\begin{array}{l}295-5-20 \\
V x-1\end{array}$ \\
\hline FACTIITy & 222-S LABORATORY \\
\hline FUNCTION & $\begin{array}{l}\text { FIHTERE AT FROM THE } \\
\text { COUNTING COOM VAUL }\end{array}$ \\
\hline
\end{tabular}

\begin{tabular}{|c|c|c|c|}
\hline 1975 & $\begin{array}{c}101 \\
12 \\
72 \\
2^{2}\end{array}$ & 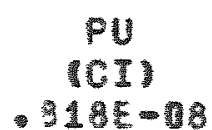 & 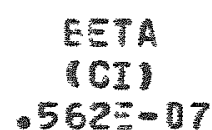 \\
\hline $\begin{array}{l}F=9 \\
=98\end{array}$ & 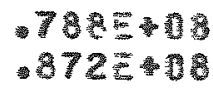 & 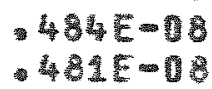 & $\begin{array}{r}529=-17 \\
-46^{=}=17\end{array}$ \\
\hline$M P$ & 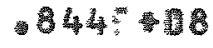 & $351=0$ & $-193=-16$ \\
\hline 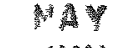 & $928 \%$ & $-623-13$ & $35=-9$ \\
\hline 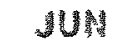 & 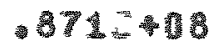 & .385 政 & 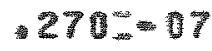 \\
\hline$\sqrt{3}$ & 67254 & 361 箱 & $-3 E z^{2}-07$ \\
\hline AUs & 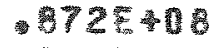 & $-378=08$ & $639=07$ \\
\hline $5=0$ & 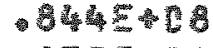 & $-664=08$ & $-558-17$ \\
\hline $0 \%$ & $-372=$ & $-55 \%-15$ & $463=07$ \\
\hline 焟) & 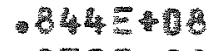 & $971 E-18$ & $-528=-7$ \\
\hline $\mathrm{S}=\mathrm{C}$ & - 325 & * & 费器 $1=-17$ \\
\hline 4.3 & & & \\
\hline
\end{tabular}

MONTHEY

\begin{tabular}{|c|c|c|c|}
\hline 管 & $.1045+10$ & $.715 E-17$ & $.6155-16$ \\
\hline $\begin{array}{l}\text { MOH THLY } \\
\text { MX }\end{array}$ & & CI/OAY & SCI/OAYY \\
\hline FELELSE & & $-324=09$ & $-343=-08$ \\
\hline $\begin{array}{l}\text { AVER CE } \\
\text { PLSESE }\end{array}$ & & $-196 c-09$ & $-167=02$ \\
\hline $\begin{array}{l}\text { ANERGE } \\
\text { Conc. }\end{array}$ & & $\begin{array}{l}\text { UUCI/CC) } \\
.691 E-13\end{array}$ & $\begin{array}{l}\text { UCI/CC } \\
589=-12\end{array}$ \\
\hline
\end{tabular}

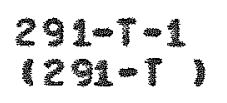

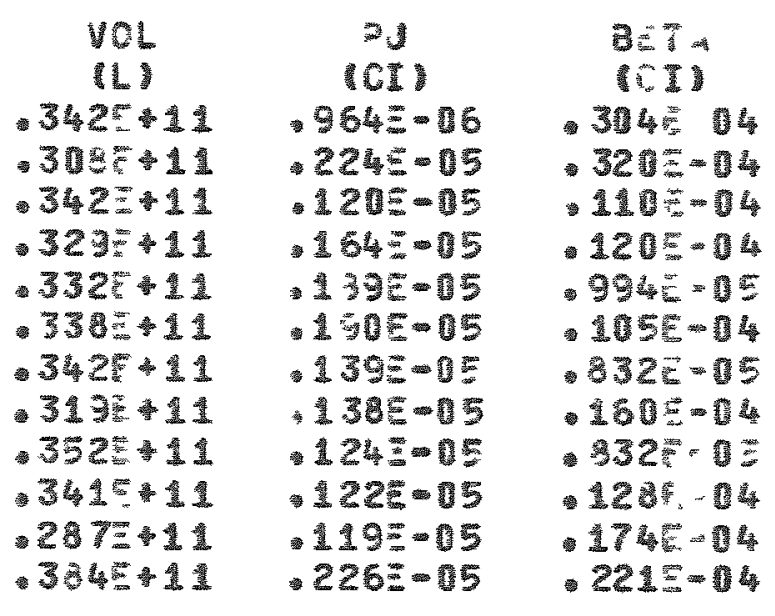

$-4025-12 \cdot 185 \pm-44.1915-13$

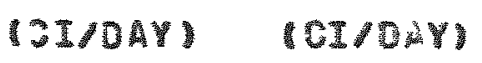

$.8005-17, \quad 114 E-05$

$.513 E=07 \quad .528 E=06$

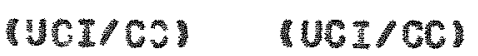

$-461=-13 \quad-475 E-12$ 
5TCK NO. $296-5=3$

FLAN CODE ITK-5

FAGILITY 242-T WASTE EVAP.

FUNCTION FILTEREC EXHAUST AIR FEOM 242-i EVAPOKATOR, VESSEL V"IY

\section{$296-T-12$}

$(\mathrm{NO}-5)$

$224-T$

FILTER:D ENHUSTES FOR 224 年 3 UTLDENG.

\begin{tabular}{|c|c|c|c|}
\hline 2975 & $\begin{array}{l}\text { VOL } \\
14 L\end{array}$ & $\begin{array}{l}\text { PU } \\
\text { SOT }\end{array}$ & $\begin{array}{l}85 T \\
\text { C CII }\end{array}$ \\
\hline 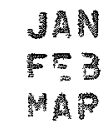 & $\begin{array}{r}453 E 09 \\
408=09 \\
453209\end{array}$ & 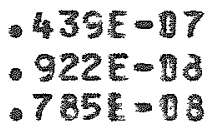 & 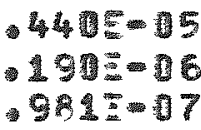 \\
\hline PD & - $23=199$ & $.599=-7$ & $-163=05$ \\
\hline 到Y & $-453=19$ & $-729=-17$ & $-978-166$ \\
\hline 武潩 & - 438 B & $-161 E-6$ & $-327=-5$ \\
\hline 110 & .45359 & $-219-06$ & $-397=05$ \\
\hline$\sqrt[1]{40}$ & $-453=09$ & -1070 & $-398=05$ \\
\hline$S=0$ & - 36509 & $-330 E-15$ & $377=-15$ \\
\hline 90 & $0453 E^{2} 009$ & -1010 & $-2285-05$ \\
\hline WOV & -45300 & $-2151-06$ & 60 \\
\hline $\mathrm{C}=\mathrm{C}$ & $-453=199$ & -1950 & $107=05$ \\
\hline
\end{tabular}

\begin{tabular}{|c|c|c|}
\hline $\begin{array}{l}\text { VOL } \\
\text { MLI }\end{array}$ & $\begin{array}{l}\mathrm{FS} \\
\mathrm{BOI}\end{array}$ & $\begin{array}{l}B+4 \\
B D 1\end{array}$ \\
\hline .687511 & $1045-05$ & 79655 \\
\hline$-618 \mathrm{E} \cdot 11$ & $911=06$ & .7750 \\
\hline 600011 & $-669=06$ & .1785 \\
\hline .7905 & $1455-05$ & 13504 \\
\hline 5725 & $.427=016$ & $5473^{2} \cdot 5$ \\
\hline $.707 E-11$ & $-123=05$ & 117504 \\
\hline . $751 E^{3}-11$ & $314=-15$ & $.576 z=05$ \\
\hline $689 \% \cdot 11$ & $.651=06$ & $.611 E=09$ \\
\hline .663511 & $-1305-05$ & $706=-0$ \\
\hline -778:-11 & $.34=-05$ & $-641 z=0$ \\
\hline$-617=11$ & $2=-16$ & \\
\hline & & \\
\hline
\end{tabular}

PONTHLY

\begin{tabular}{|c|c|c|c|}
\hline TOTALS & - 5311 & $.153 t=05$ & $-313=-64$ \\
\hline MONTHLY & & COI/CAYI & (CIPOAY) \\
\hline MXII UM & & & \\
\hline FSLSAS & & $.1100-07$ & $.2015-76$ \\
\hline $\begin{array}{l}\text { AVIOLE } \\
\text { FFL }\end{array}$ & & $.419 E=88$ & $.8573=07$ \\
\hline $\begin{array}{l}\text { AVEFAGE } \\
\text { CONC. }\end{array}$ & & $\begin{array}{l}\text { UCI/CCI } \\
-288=12\end{array}$ & $\begin{array}{l}\text { UCI/CC) } \\
.5695-11\end{array}$ \\
\hline
\end{tabular}

\begin{tabular}{|c|c|c|}
\hline \multirow[t]{5}{*}{$-83=12$} & $169 E=04$ & - $101 E=D 3$ \\
\hline & (CI/OAY) & $(C D / C A Y)$ \\
\hline & $128 E-10$ & $.574 E-06$ \\
\hline & - 462:=07 & .2775 \\
\hline & $\begin{array}{l}\operatorname{UCI} / \mathrm{CO} \\
203 \mathrm{E}-13\end{array}$ & $\begin{array}{l}\text { UCI/GCI } \\
-1215 \quad 12\end{array}$ \\
\hline
\end{tabular}




\begin{abstract}
STAK NO. 296- -13
PLAMT COCE $\quad 221-\frac{T}{2}$
\end{abstract}

FACILITYN T PLANT
FUNCION VENTLATION AIR

\begin{tabular}{|c|c|c|c|}
\hline 1975 & $\mathrm{VOL}$ & PU & Be \\
\hline & (b) & (CI) & CII \\
\hline 绍 & -427E事1 & $-576 E-07$ & $-559 t=06$ \\
\hline E. 9 & $.586 \div 11$ & $.6095=17$ & $.786=-06$ \\
\hline sing & $.568 \equiv-11$ & $.976 E-17$ & $125-05$ \\
\hline $\operatorname{mos}$ & 6040.51 & $-1776-65$ & $360=-14$ \\
\hline 跑Y & .18070 & $1675-06$ & $103=0$ \\
\hline 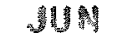 & $.53 E+1$ & $.545 E-06$ & $6 E-75$ \\
\hline UL & -719\%-11 & $.125 E-05$ & -05 \\
\hline aug & 35 & 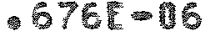 & \\
\hline$\xi=0$ & 3777 & $748-06$ & $=0$ \\
\hline 0? & $-3325+11$ & - $585 E-06$ & $=0$ \\
\hline Mov & $.8578=14$ & - 145E=05 & $-105=0$ \\
\hline PEc & - 8245 & $.577 E-16$ & $-471=-55$ \\
\hline
\end{tabular}

MOHWH

\begin{tabular}{|c|c|c|c|}
\hline Tor als & 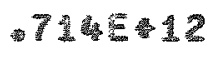 & -90015 & $.9035-04$ \\
\hline 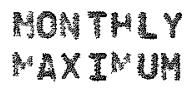 & & VIVDAY & SCIDAY \\
\hline PELESSE & & $632=07$ & $-229 E=05$ \\
\hline $\begin{array}{l}\text { AVE OA GE } \\
\text { PELEASE }\end{array}$ & & $-2371-7$ & $-268 E-16$ \\
\hline $\begin{array}{l}\text { MVERGE } \\
\text { CONS. }\end{array}$ & & $\begin{array}{l}\text { IUCT/CC } \\
.121-13\end{array}$ & $\begin{array}{l}\text { PUCI/CC } \\
126=12\end{array}$ \\
\hline
\end{tabular}

\section{$296-T-16$ \\ 12糸2}

242-TA VMULT

FILTE 242-T、 VAULT AND FROM 242-落 107 CELS.

\begin{tabular}{|c|c|c|}
\hline 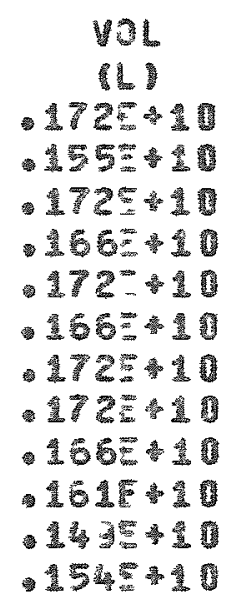 & 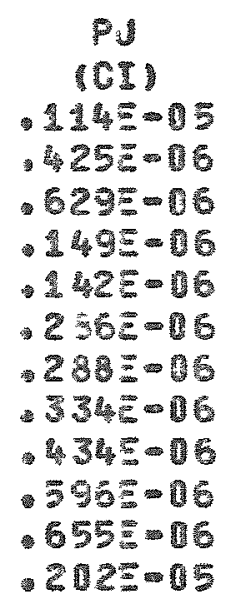 & 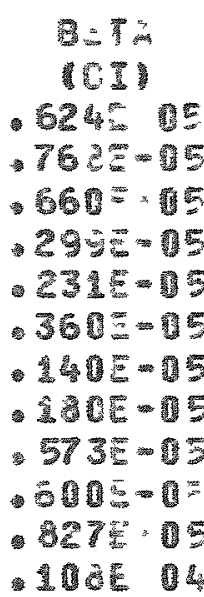 \\
\hline
\end{tabular}

\begin{tabular}{|c|c|c|}
\hline \multirow[t]{5}{*}{ 198E些1 } & $.709=-05$ & $.535 E=04$ \\
\hline & (EIODY) & (OD/OEY) \\
\hline & $.6505=07$ & - $3505-6$ \\
\hline & $194=07$ & 174506 \\
\hline & $\begin{array}{l}\text { UCI/CO1 } \\
-359=12\end{array}$ & $\begin{array}{l}14 C I / 0 C 1 \\
-321 E=11\end{array}$ \\
\hline
\end{tabular}


STACK NO. $296-T-17$
PLAMT COCE $1 \mathrm{K2}-1.1$

FACILITY 242-T WASTE EVAP.

FUNCTION FILTEREE EXHAUST AIR FPOM THE EVAPOPATOR AND COLO CELL.

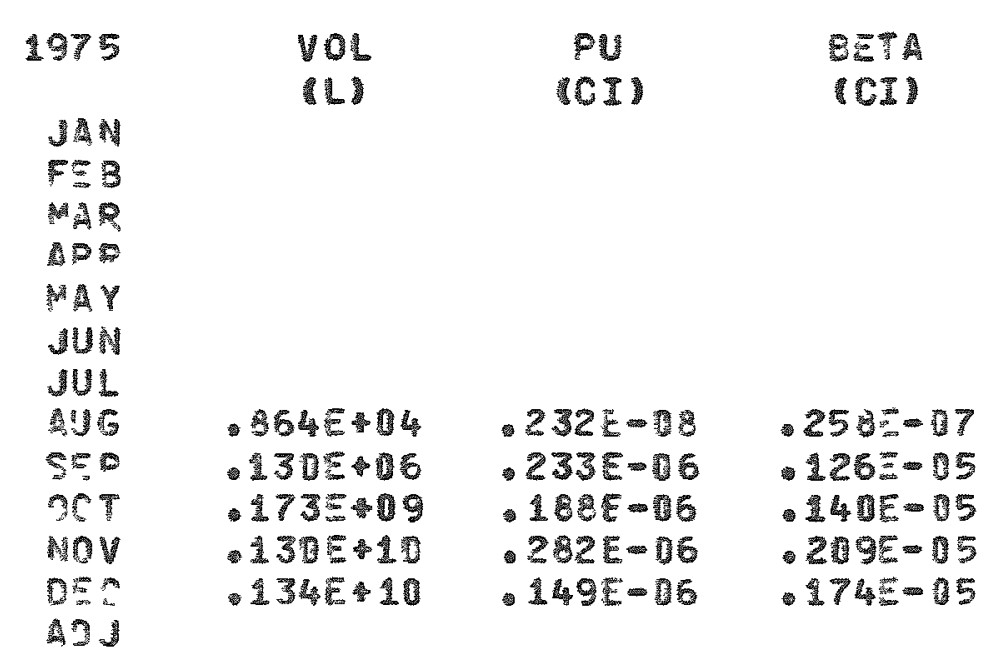

MONTHLY

\begin{tabular}{|c|c|c|}
\hline TOTALS 281E+1D & $.854 E-15$ & $.651=05$ \\
\hline $\begin{array}{l}\text { MOATHL } \\
\text { MA X IMUY }\end{array}$ & TCIOAY) & (CIODY) \\
\hline PELER SE & $.940 E-08$ & $.696 E-07$ \\
\hline $\begin{array}{l}\text { AYPOGE } \\
\text { FFL SASE }\end{array}$ & $.595 E-08$ & $.529=-07$ \\
\hline $\begin{array}{l}\text { AVEPAGE } \\
\text { CONC. }\end{array}$ & $\begin{array}{l}\text { UCI/CCI } \\
.374-12-12\end{array}$ & $\begin{array}{l}\text { UCI/CC } \\
232 z-11\end{array}$ \\
\hline
\end{tabular}

$291-U-1$ 291-U

4 PLAN

FILTERED AIR FR3 221-U CAMYON VENTILLATION ANO HOT SIOE OF 241-WP VAULT.

\begin{tabular}{|c|c|c|}
\hline 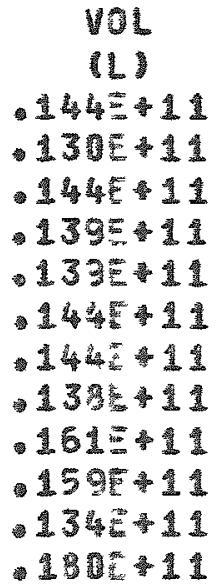 & 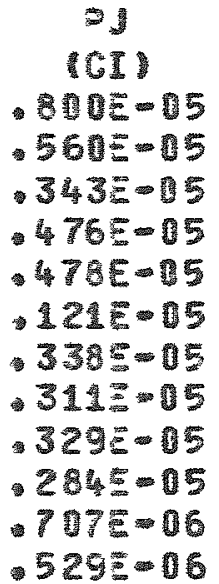 & 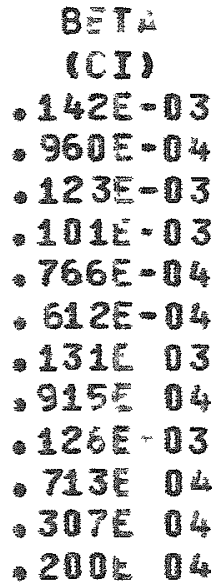 \\
\hline
\end{tabular}

\begin{tabular}{|c|c|c|}
\hline \multirow[t]{5}{*}{$176 * 12$} & $-455=04$ & $107=02$ \\
\hline & $107 / O A Y 1$ & (CI/DAY) \\
\hline & $-258=-16$ & $-458-15$ \\
\hline & - 195E-06 & $.297 E-05$ \\
\hline & $\begin{array}{l}\text { UCI/Oz) } \\
.237=-12\end{array}$ & $\begin{array}{l}\text { (UCI/CC) } \\
-610^{2}-11\end{array}$ \\
\hline
\end{tabular}




\begin{tabular}{|c|c|}
\hline $\begin{array}{l}\text { STACK NO: } \\
\text { OLAT CODE }\end{array}$ & $\begin{array}{l}296=0-10 \\
271-1)\end{array}$ \\
\hline FACILITY & 271-U UU STCFAGE \\
\hline FUNO ION & 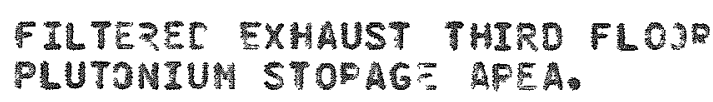 \\
\hline
\end{tabular}

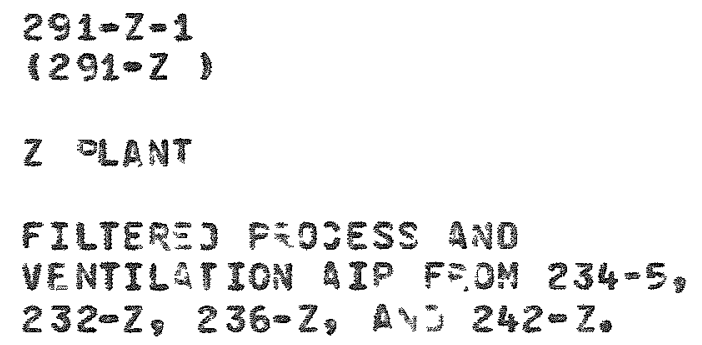

\begin{tabular}{|c|c|c|c|}
\hline 1975 & $\begin{array}{l}\text { YOL } \\
\text { gL }\end{array}$ & PU & $\begin{array}{l}\text { BETA } \\
\text { (GTY }\end{array}$ \\
\hline 结期 & 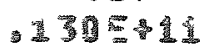 & $.397 E=17$ & $.359=-06$ \\
\hline $5=3$ & 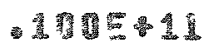 & $-280=-07$ & $.283=06$ \\
\hline $7 x^{2}$ & - 104 & .281:-47 & $-268=-16$ \\
\hline IPR & $-130=-11$ & $-105=06$ & $-3855-6$ \\
\hline 期 & 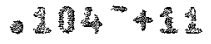 & $475-107$ & $-252=-06$ \\
\hline 列期 & 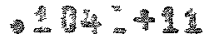 & $396-7=7$ & $5505-05$ \\
\hline WUL & $130=11$ & $.7938 \times 7$ & $.531=16$ \\
\hline Allo & $.357=10$ & $\cdot 455=7$ & $.211-96$ \\
\hline $5=9$ & & & \\
\hline \multicolumn{4}{|l|}{ act } \\
\hline \multicolumn{4}{|l|}{ Mov } \\
\hline \multicolumn{4}{|l|}{$n=c$} \\
\hline \multicolumn{4}{|l|}{ GDS } \\
\hline \multicolumn{4}{|l|}{ MonTHEY } \\
\hline TOFALS & 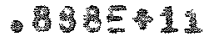 & $-45 c-16$ & $.235=5$ \\
\hline MOHTHLY & & RCI/CAYU & CI/SOH \\
\hline$M X I N U M$ & & & \\
\hline FELEASF & & $-3000-08$ & $196=-7$ \\
\hline \multicolumn{4}{|l|}{ AN 36} \\
\hline PELEAS & & $-1748=88$ & 1995 \\
\hline AVPRAGE & & TUCI/COI & $\operatorname{PUCI} / 0 \mathrm{OC}$ \\
\hline CONG. & & - 467 7 & - 322 \\
\hline
\end{tabular}

\begin{tabular}{|c|c|c|}
\hline \multirow[t]{5}{*}{. 3535} & $.295=-3$ & $.203=-02$ \\
\hline & 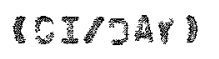 & $(2 I / D-Y)$ \\
\hline & - $198=05$ & $1015-04$ \\
\hline & $-616=06$ & $.564 E=05$ \\
\hline & $\begin{array}{l}\text { UCI/CB } \\
-835=13\end{array}$ & $\begin{array}{l}\text { UOT/CCI } \\
.5775-32\end{array}$ \\
\hline
\end{tabular}


STACK NO: $\quad 296-2-3$

$241-2$

\section{FACILITY $241=2$ VAULT}

FUNCTION FILTERED ATR FOM $241-Z$ SUMP HO VESSEL VENTS.

\begin{tabular}{|c|c|c|c|}
\hline 1975 & $\begin{array}{l}110 L \\
115\end{array}$ & $\begin{array}{c}P U \\
\text { CE }\end{array}$ & $\begin{array}{l}987 A \\
19 T 1\end{array}$ \\
\hline JAN & $177=10$ & $-2505-06$ & 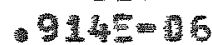 \\
\hline FE & $-160 \leq-10$ & $-1445-15$ & - 113E=0 \\
\hline $\begin{array}{l}\text { MAO } \\
\text { POO }\end{array}$ & $\begin{array}{l}175+10 \\
-1655-10\end{array}$ & $\begin{array}{l}140 t-126 \\
157 t=16\end{array}$ & $\begin{array}{r}104 E-05 \\
171 E-15\end{array}$ \\
\hline Mu & $1745+10$ & $-5095-16$ & $-828=05$ \\
\hline WU & $-1725+10$ & $.199 E-06$ & $6124=06$ \\
\hline UL & $-176 E+1$ & $-215 E-06$ & $115=-15$ \\
\hline 1ug & 177510 & $.8735=07$ & $.673 E-06$ \\
\hline$S p$ & $1718+10$ & $-122 E-16$ & $.8215-46$ \\
\hline Co? & 1775 & $.1036-76$ & $.885=-6$ \\
\hline MOV & $-171=10$ & $.933=07$ & $.576=06$ \\
\hline DES & 177510 & $372=-17$ & $560-6$ \\
\hline
\end{tabular}

MONTHLY

\begin{tabular}{|c|c|c|c|}
\hline TOTALS & $.207=-11$ & $.340 E-05$ & $-2065-04$ \\
\hline MONTHY & & ICISCAV & ICIOAM \\
\hline MX & & & \\
\hline GELESSE & & $.514 E-17$ & $-4045=6$ \\
\hline $\begin{array}{l}\text { AV: } \\
\text { PELELSE }\end{array}$ & & $.9405-03$ & $568=07$ \\
\hline $\begin{array}{l}\text { AVEREG } \\
\text { CONC. }\end{array}$ & & 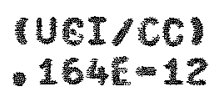 & $\begin{array}{l}\text { UCI/CO } \\
-992-12\end{array}$ \\
\hline
\end{tabular}

$295-Z=9$

Z9STK

$216-2=9$ CRE

FILTEREO MIR FROM THF $216=2=9$ CRTB ANO CUBTCLE

\begin{tabular}{|c|c|}
\hline 10L & DU \\
\hline
\end{tabular}

\begin{tabular}{|c|c|c|}
\hline $\begin{array}{l}.735+08 \\
236=10\end{array}$ & $\begin{array}{l}-260=-17 \\
\cdot 354=-16\end{array}$ & $\begin{array}{l}-1725-17 \\
-128=05\end{array}$ \\
\hline $160 E+12$ & $.884=06$ & $.3015 \cdot 0=$ \\
\hline \multirow[t]{5}{*}{$163 E+12$} & $-126 \bar{E}-05$ & $-4305-05$ \\
\hline & (SIOAY & $(0 T / D A Y)$ \\
\hline & $-260=-07$ & 473 年 07 \\
\hline & $1368-07$ & .154606 \\
\hline & $\begin{array}{l}\text { (UCI/CS) } \\
-776 E=14\end{array}$ & $\begin{array}{l}\text { UCD/CCU } \\
-26,4 E-13\end{array}$ \\
\hline
\end{tabular}




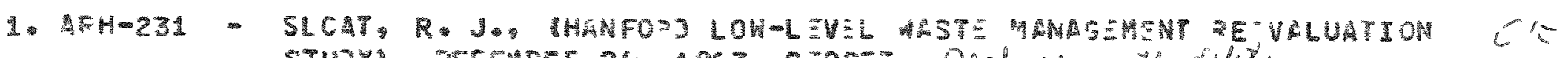

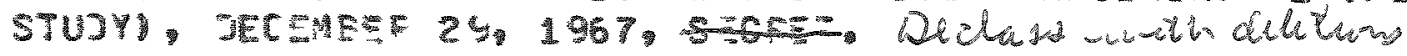

2. 35 粗-968

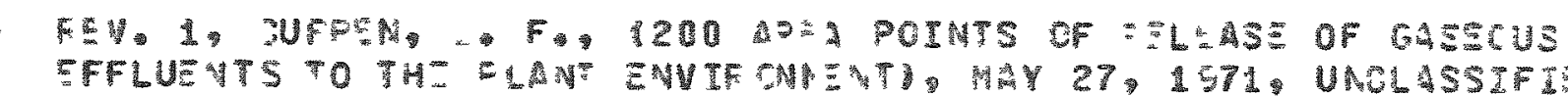

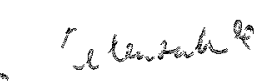

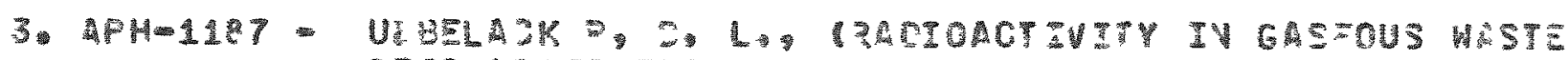

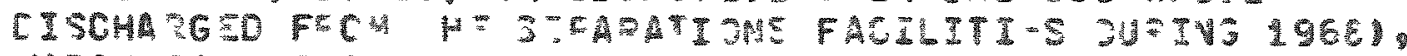

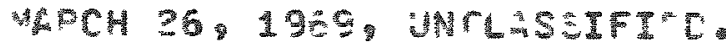

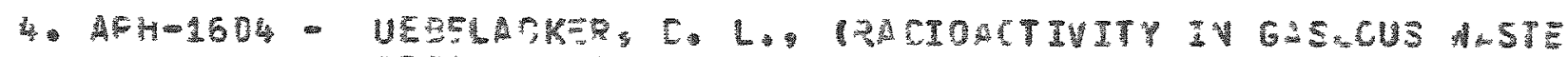

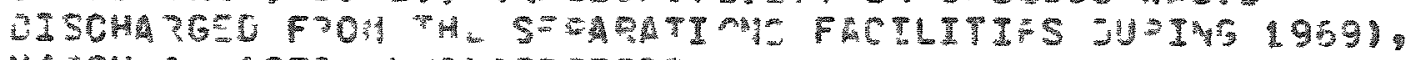

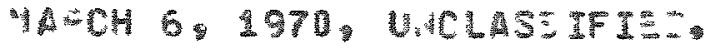

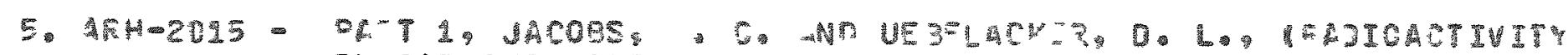

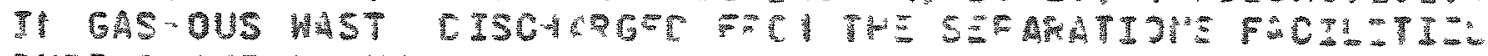

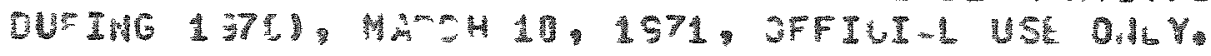

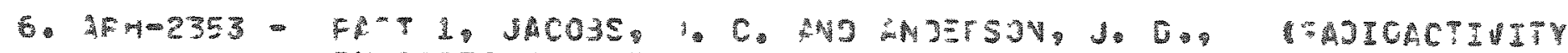

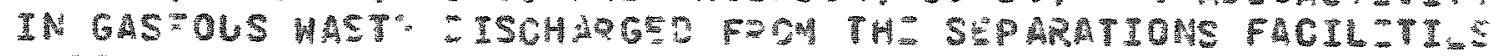

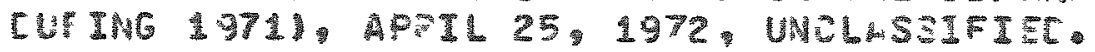

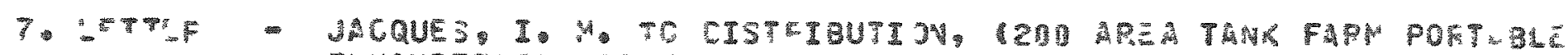

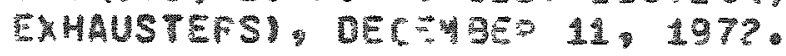

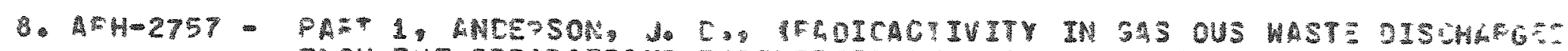

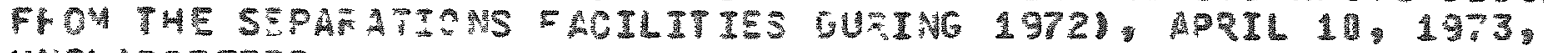
UNGLASSIFIED.

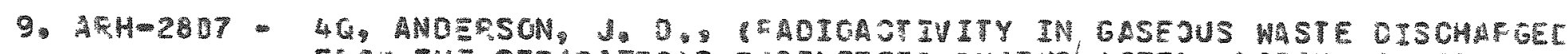

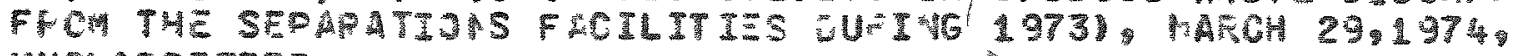
UN:OLSSIFTED.

10. ARH-3C94 - 40 ANO

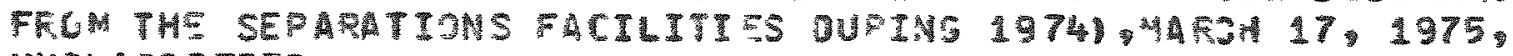
UNCLASSIFIFD. 